\title{
Current understanding of the driving mechanisms for spatiotemporal vari- ations of atmospheric speciated mercury: a review
}

\section{Huiting Mao et al.}

Correspondence to: Huiting Mao (hmao@esf.edu)

The copyright of individual parts of the supplement might differ from the CC-BY 3.0 licence. 
Table S1. Marine boundary layer TGM or GEM ( $\mathrm{ng} \mathrm{m}^{-3}$; units are noted otherwise). Units were converted in STP. The units for time resolution are hours (denoted as h), minutes (denoted as min), and seconds (denoted as s). GEM measurements are indicated with a superscript G.

\begin{tabular}{|c|c|c|c|c|c|c|c|}
\hline & Platform & Latitude & Sampling Period & $\begin{array}{l}\text { Time } \\
\text { Resolution }\end{array}$ & Range & Mean $( \pm \sigma)$ & Reference \\
\hline \multirow[t]{14}{*}{ Atlantic } & Ship & $54^{\circ} \mathrm{N}, 10^{\circ} \mathrm{E}-20^{\circ} \mathrm{N}, 67^{\circ} \mathrm{W}$ & Oct - Nov 1973 & $2-4-h$ & $1-10$ & 2.8 & Seiler et al. (1980) \\
\hline & Ship & $35^{\circ} \mathrm{S}-11^{\circ} \mathrm{N}$ & $1977-1980$ & 40-min & $0.8-2.09$ & $1.31 \pm 0.21$ & Slemr et al. $(1981,1985)$ \\
\hline & Ship & $11^{\circ} \mathrm{N}-54^{\circ} \mathrm{N}$ & $1977-1980$ & $20-40-\min$ & $1.0-3.41$ & $1.96 \pm 0.35$ & Slemr et al. $(1981,1985)$ \\
\hline & Ship & $\begin{array}{l}33.36^{\circ} \mathrm{S}-8.41^{\circ} \mathrm{N}, 20.23^{\circ} \mathrm{W} \\
-51.55^{\circ} \mathrm{W}\end{array}$ & 16 May - 20 Jun 1996 & 24-h & $1.17-1.99$ & $1.61 \pm 0.09$ & Lamborg et al. (1999) \\
\hline & Ground $^{\mathrm{G}}$ & Chesapeake Biol. Lab & $1997-1999$ & $6-24-h$ & $1.03-2.5$ & $1.83 \pm 0.43$ & Sheu and Mason (2001) \\
\hline & Ship & $\begin{array}{l}\text { Near Bermuda } \\
\left(31^{\circ} 40^{\prime} \mathrm{N}, 64^{\circ} 10^{\prime} \mathrm{W}\right)\end{array}$ & Sep, Dec 1999, Mar 2000 & N/A & $\begin{array}{l}6.5-10.95 \mathrm{pmol} \\
\mathrm{m}^{-3}(1.3-2.2)\end{array}$ & $\begin{array}{l}9.91 \pm 2 \mathrm{pmol} \mathrm{m}^{-3} \\
(2 \pm 0.4)\end{array}$ & Mason et al. (2001) \\
\hline & Ground & $\begin{array}{l}\text { Mace Head }\left(53^{\circ} 20^{\prime} \mathrm{N},\right. \\
\left.9^{\circ} 54^{\prime} \mathrm{W}\right)\end{array}$ & $\begin{array}{l}\text { Sep } 1995 \text { - Dec } 2001 \\
1996-2013\end{array}$ & 5-min & & $\begin{array}{l}1.72 \\
1.663 \pm 0.16 \\
\end{array}$ & $\begin{array}{l}\text { Ebinghaus et al. (2002) } \\
\text { Weilgelt et al. (2015) }\end{array}$ \\
\hline & Ship & $\begin{array}{l}67^{\circ} \mathrm{N}-71^{\circ} \mathrm{S} \\
\text { Neumayer - Punta } \\
\text { Arenas }\end{array}$ & $\begin{array}{l}\text { Oct - Nov } 1996 \\
\text { Dec } 1999-\text { Jan } 2000 \\
\text { Feb } 2001\end{array}$ & $\begin{array}{l}\text { 30-min } \\
15-\min \\
5-\min \end{array}$ & $\begin{array}{l}0.76-1.84 \\
0.27-2.34\end{array}$ & $\begin{array}{l}1.26 \pm 01 \\
1.08 \pm 0.29 \\
1.1 \pm 0.2 \\
\end{array}$ & $\begin{array}{l}\text { Temme et al. (2003a) } \\
\text { Temme et al. (2003b) }\end{array}$ \\
\hline & Ground $^{\mathrm{G}}$ & Pompano Beach, FL & Jun 2000 & 5-min & $<1.8$ & $1.6 \pm 0.06$ & Malcom et al. (2003) \\
\hline & Ship $^{G}$ & $\begin{array}{l}54^{\circ} \mathrm{N}-85^{\circ} \mathrm{N} \\
78^{\circ} \mathrm{N}-85^{\circ} \mathrm{N} \\
\end{array}$ & 16 Jun - 29 Aug 2004 & 5 -min & $\begin{array}{l}1.15-1.95 \\
1.17-2.72 \\
\end{array}$ & $\begin{array}{l}1.53 \pm 0.12 \\
1.82 \pm 0.24\end{array}$ & Aspmo et al. (2006) \\
\hline & Ship $^{\mathrm{G}}$ & $\begin{array}{l}\text { Norfolk, VA to Bermuda to } \\
\text { Barbados }\end{array}$ & 15 Aug - 8 Sep 2003 & 5-min & $1.55-1.9$ & $1.63 \pm 0.08$ & Laurier et al. (2007) \\
\hline & Ground & Cape Point, South Africa & $\begin{array}{l}1996-2004 \\
\text { Mar 2007- Dec } 2007\end{array}$ & 15-min & $\begin{array}{l}0.49-6.78 \\
<0.05-5.44^{\mathrm{G}}\end{array}$ & $\begin{array}{l}1.18 \pm 0.11 \sim 1.52 \pm 0.37 \\
0.94 \pm 0.16\end{array}$ & $\begin{array}{l}\text { Slemr et al. (2008) } \\
\text { Brunke et al. (2010) }\end{array}$ \\
\hline & Ship $^{G}$ & $\begin{array}{l}58^{\circ} \mathrm{N}-67^{\circ} \mathrm{N} \\
43^{\circ} \mathrm{N}-59^{\circ} \mathrm{N} \\
23^{\circ} \mathrm{N}-45^{\circ} \mathrm{N} \text { Sargasso Sea } \\
39^{\circ} \mathrm{S}-3^{\circ} \mathrm{S} \quad \text { South Africa }\end{array}$ & $\begin{array}{l}\text { 16 Aug - 1Sep } 2006 \\
15-24 \text { Apr } 2007 \\
30 \text { Mar }-11 \text { Apr } 2007 \\
8-21 \text { Oct } 2006\end{array}$ & 5-min & & $\begin{array}{l}1.32 \pm 0.16 \\
2.26 \pm 0.26 \\
2.86 \pm 0.17 \\
1.36 \pm 0.24\end{array}$ & Soerensen et al. (2010) \\
\hline & Ground & Appledore Island, ME, US & Jun 2007 - Aug 2010 & 5-min & $\begin{array}{l}<3251 \mathrm{ppqv} \\
(\sim 29)\end{array}$ & $\begin{array}{l}147 \pm 29 \mathrm{ppqv} \\
(1.31 \pm 0.26)\end{array}$ & Mao et al. (2012) \\
\hline \multirow[t]{5}{*}{ Indian } & Ship & $\begin{array}{l}39^{\circ} \mathrm{S}-33^{\circ} \mathrm{S} \\
22^{\circ} \mathrm{S}-17^{\circ} \mathrm{S} \text { West Australia }\end{array}$ & $\begin{array}{l}22-29 \text { Oct } 2006 \\
3-6 \text { Nov } 2006\end{array}$ & 5-min & & $\begin{array}{l}1.11 \pm 0.19 \\
1.03 \pm 0.16\end{array}$ & Soerensen et al. (2010) \\
\hline & Ship & Eq . - Prydz Bay, Antarctica & 24 Nov - 11 Dec 2007 & $5-\min$ & $0.3-4.496$ & $1.471 \pm 0.842$ & Xia et al. (2010) \\
\hline & Ship & $9^{\circ} \mathrm{S}-21^{\circ} \mathrm{S}$ & Nov 2007 & $15-30-\min$ & $1.05-1.51$ & 1.2 & Witt et al. (2010) \\
\hline & Ground & $\begin{array}{l}\text { Nieuw Nickerie } 5^{\circ} 56^{\prime} \mathrm{N}, \\
56^{\circ} 59^{\prime} \mathrm{W}\end{array}$ & 2007 & N/A & N/A & $\begin{array}{l}\text { NH: } 1.450 \pm 0.268 \\
\text { SH: } 1.324 \pm 0.225\end{array}$ & Müller et al. (2012) \\
\hline & Ground $^{\mathrm{G}}$ & $\begin{array}{l}\text { Amsterdam Island } 37^{\circ} 48^{\prime} \mathrm{S} \text {, } \\
77^{\circ} 34^{\prime} \mathrm{E}\end{array}$ & Jan 2012 to Dec 2013 & 5-min & $0.72-1.55$ & $1.03 \pm 0.03$ & Angot et al. (2014) \\
\hline Pacific & Ground & San Francisco Bay & $1965-1966$ & 9-min & $1-2$ & & Williston (1968) \\
\hline
\end{tabular}




\begin{tabular}{|c|c|c|c|c|c|c|c|}
\hline & Airborne & $\begin{array}{l}60^{\circ} \mathrm{N}-55^{\circ} \mathrm{S}, 8-10 \mathrm{~km} \\
\text { altitudes }\end{array}$ & 1973 & $2-4-h$ & $0.6-2.5$ & 1.5 & Seiler et al. (1980) \\
\hline & Ship & $15^{\circ} \mathrm{N}-20^{\circ} \mathrm{S}, \sim 160^{\circ} \mathrm{W}$ & Oct 1980 & N/A & $\begin{array}{l}1.0-2.25 \\
4^{\circ} \mathrm{N}-10^{\circ} \mathrm{S}: 1.5- \\
2.25\end{array}$ & $\begin{array}{l}\text { N. of } 10^{\circ} \mathrm{N}: 1.56 \\
\text { S. } 10^{\circ} \mathrm{S}: 1.0\end{array}$ & Fitzgerald et al. (1984) \\
\hline & Ship & $\begin{array}{l}4.5^{\circ} \mathrm{N}-4.5^{\circ} \mathrm{S} \text { at } 85^{\circ} \mathrm{W} ; \\
155^{\circ} \mathrm{W}-93^{\circ} \mathrm{W} \text {, in the equ. }\end{array}$ & 8 June - 3 July 1984 & N/A & $0.85-1.13$ & $1.02 \pm 0.08$ & Kim \& Fitzgerald (1986) \\
\hline & Ground & $48.3 \mathrm{~N}, 124.6 \mathrm{~W}, 480 \mathrm{~m}$ asl & May 2001 - May 2002 & 5-min & N/A & $\begin{array}{l}\text { Spr: } 1.54 \pm 0.16 \\
\text { Sum: } 1.61 \pm 0.13 \\
\text { Fall: } 1.54 \pm 0.09 \\
\text { Win: } 1.51 \pm 0.12\end{array}$ & Weiss-Penzias et al. (2003) \\
\hline & Ship $^{G}$ & $\begin{array}{l}\text { Osaka, Japan - Honolulu, } \\
\text { Hawaii }\end{array}$ & 1 May $2002-4$ Jun 2002 & 5 -min & $1.6-4.7$ & 2.5 & Laurier et al. (2003) \\
\hline & Airborne $^{\mathrm{G}}$ & $35^{\circ} \mathrm{N}-55^{\circ} \mathrm{N}, 0-8 \mathrm{~km}$ alt. & Spring 2002 & $2.5-\mathrm{min}$ & $0.5-1.2$ & N/A & Radke et al. (2007) \\
\hline & Ground $^{\mathrm{G}}$ & $26.8^{\circ} \mathrm{N}, 128.2^{\circ} \mathrm{E}, 60 \mathrm{~m}$ amsl & 23 Mar - 2 May 2004 & 5 -min & $1.5-5$ & $2.04 \pm 0.38$ & Chand et al. (2008) \\
\hline & Ship & Shanghai, China - Eq. & 12 Nov - 17 Dec 2007 & $5-\min$ & $1.075-3.698$ & $1.746 \pm 0.513$ & Xia et al. (2010) \\
\hline & Ship $^{G}$ & $\begin{array}{l}44^{\circ} \mathrm{S}-26^{\circ} \mathrm{S} \text { E. Australia } \\
27^{\circ} \mathrm{S}-7^{\circ} \mathrm{S} \text { Coral Sea } \\
56^{\circ} \mathrm{S}-26^{\circ} \mathrm{S} \text { New Zealand } \\
58^{\circ} \mathrm{S}-33^{\circ} \mathrm{S} \text { Chilean Coast }\end{array}$ & $\begin{array}{l}23 \text { Nov - 15 Dec } 2006 \\
16 \text { Dec } 2006-3 \text { Jan } \\
2007 \\
3-14 \text { Jan } 2007 \\
31 \text { Jan }-8 \text { Feb } 2007\end{array}$ & $5-\min$ & & $\begin{array}{l}1.33 \pm 0.24 \\
1.21 \pm 0.18 \\
1.19 \pm 0.17 \\
1.11 \pm 0.11\end{array}$ & Soerensen et al. (2010) \\
\hline & Ground $^{\mathrm{G}}$ & $\begin{array}{l}\text { Mt Bachelor, Oregon, USA, } \\
43.981^{\circ} \mathrm{N} \\
121.691^{\circ} \mathrm{W} 2.7 \mathrm{~km} \text { a.s.l. }\end{array}$ & $2005-2011$ & 5-min & $1.2-1.4$ & N/A & Timonen et al. (2013) \\
\hline & Ship & $30^{\circ} \mathrm{N}-60^{\circ} \mathrm{N}$ & Jul - Sep 2008 & 5-min & $0.30-6.02$ & $1.52 \pm 0.68$ & Kang\&Xie (2011) \\
\hline & Ship $^{G}$ & $\begin{array}{l}\text { San Diego - San Francisco, } \\
\text { CA }\end{array}$ & 14 May - 8 Jun 2010 & $5-\min$ & $<7.21$ & 1.41 & Weiss-Penzias et al. (2013) \\
\hline & Ground $^{\mathrm{G}}$ & $\begin{array}{l}\text { Puerto Villamil, Isabela } \\
\left.\text { Island ( } 0^{\circ} 57^{\prime} \text { S, } 90^{\circ} 58^{\prime} \mathrm{W}\right) \\
\text { Puerto Baquerizo Moreno, } \\
\text { San Cristóbal Island, } \\
0^{\circ} 54^{\prime} \text { 'S, 89³6' W }\end{array}$ & $\begin{array}{l}\text { Feb - Jun } 2011 \\
\text { Jul - Oct } 2011\end{array}$ & 5-min & $0.58-2$ & $1.08 \pm 0.17$ & Wang et al. (2014) \\
\hline & Ship $^{G}$ & $20^{\circ} \mathrm{N}$ to $15^{\circ} \mathrm{S}$ Samoa - HW & $1-24$ October 2011 & 5-min & N/A & $\begin{array}{l}14-20^{\circ} \mathrm{N} 1.32 \pm 0.05 \\
\mathrm{ITCZ}^{\circ}-14^{\circ} \mathrm{N} 1.27 \pm 0.10 \\
\text { Equ. } 1^{\circ} \mathrm{S}-5^{\circ} \mathrm{N} 1.18 \pm 0.05 \\
1-15^{\circ} \mathrm{S} 1.15 \pm 0.05\end{array}$ & Soerensen et al. (2014) \\
\hline Japan Sea & Ship & $34^{\circ} \mathrm{N}-39^{\circ} \mathrm{N}$ & $\begin{array}{l}13-15 \text { Jul } \\
18-20 \text { Sep } 2008\end{array}$ & 5-min & $\begin{array}{l}0.30-1.59 \\
0.70-2.24\end{array}$ & $\begin{array}{l}0.80 \pm 0.36 \\
1.14 \pm 0.23\end{array}$ & Kang\&Xie (2011) \\
\hline Okhotsk Sea & Ship & $45^{\circ} \mathrm{N}-56^{\circ} \mathrm{N}$ & $\begin{array}{l}15-18 \text { Jul } \\
13-18 \text { Sep } 2008\end{array}$ & 5 -min & $\begin{array}{l}0.37-1.19 \\
0.52-2.88\end{array}$ & $\begin{array}{l}0.74 \pm 0.15 \\
1.71 \pm 0.43\end{array}$ & Kang\&Xie (2011) \\
\hline Bering Sea & Ship & $58^{\circ} \mathrm{N}-66^{\circ} \mathrm{N}$ & $\begin{array}{l}18 \text { Jul }-1 \text { Aug } \\
9-13 \text { Sep } 2008\end{array}$ & 5-min & $\begin{array}{l}0.61-6.02 \\
0.55-3.07\end{array}$ & $\begin{array}{l}1.94 \pm 0.85 \\
1.60 \pm 0.46\end{array}$ & Kang\&Xie (2011) \\
\hline
\end{tabular}




\begin{tabular}{|c|c|c|c|c|c|c|c|}
\hline $\begin{array}{l}\text { South China } \\
\text { Sea }\end{array}$ & Ship & Northern SCS & 10-28 Aug 2007 & $5-\min$ & $1.04-6.57$ & 2.62 & Fu et al. (2010b) \\
\hline \multirow[t]{3}{*}{ Yellow Sea } & Ship & $\begin{array}{l}\text { Incheon - Jeju } \\
\text { Incheon - Qingdao } \\
\text { Incheon - Weihai }\end{array}$ & $\begin{array}{l}\text { 10-14 Sep } 2007 \\
\text { 16-20 Oct } 2007 \\
28 \text { Apr - 01 May } 2008\end{array}$ & $30 \mathrm{~s}$ & $\begin{array}{l}1.56-2.97 \\
1.29-2.36 \\
1.36-1.99 \\
\end{array}$ & $\begin{array}{l}2.43 \pm 0.59 \\
1.82 \pm 0.51 \\
2.03 \pm 0.66 \\
\end{array}$ & Nyugen et al. (2011) \\
\hline & Ship $^{\mathrm{G}}$ & Western and central & 9-18 Jul 2010 & $1 \mathrm{~min}$ & $1.68-4.34$ & $2.61 \pm 0.5$ & Ci et al. (2011) \\
\hline & Ship $^{G}$ & & $\begin{array}{l}\text { May } 2003-\text { Dec } 2005 \\
\text { Winter } \\
\text { Summer } \\
\text { Spring } \\
\text { Fall } \\
\end{array}$ & 15-min - hours & $\begin{array}{l}4-6 \\
2-3 \\
\text { N/A } \\
\text { N/A } \\
\end{array}$ & $\begin{array}{l}5.7 \pm 0.2 \\
2.8 \pm 0.2 \\
3.3 \pm 0.9 \\
3.8 \pm 0.3 \\
\end{array}$ & Tseng et al. (2012) \\
\hline \multirow[t]{2}{*}{$\begin{array}{l}\text { Mediterranea } \\
\mathrm{n} \text { Sea }\end{array}$} & Ground & $\begin{array}{l}\text { Palma de } \\
\text { Mallorca }\end{array}$ & 13 Nov - 6 Dec 1998 & 5-min (Tekran) & $2.4-6.19$ & $3.67 \pm 0.87$ & Pirrone et al. (2003) \\
\hline & Ship & Western and eastern sectors & 14 Jul - 9 Aug 2000 & 5-min & $0.4-11.2$ & $1.9 \pm 1.02$ & Sprovieri et al. (2003) \\
\hline Dead Sea & Ground $^{\mathrm{G}}$ & $\begin{array}{l}\text { Ein Bokek, Israel } 31.20^{\circ} \mathrm{N}, \\
35.37^{\circ} \mathrm{E}, 423 \text { bsl }\end{array}$ & $\begin{array}{l}\text { 29 Jun - 28 Jul 2009; } 29 \\
\text { Dec } 2009-17 \text { Jan } 2010 \\
\end{array}$ & 5-min & $22-450$ ppqv & N/A & $\begin{array}{l}\text { Obrist et al. (2011); Moore } \\
\text { et al. (2013) }\end{array}$ \\
\hline \multirow[t]{2}{*}{ Adriatic } & Ship $^{G}$ & $\begin{array}{l}\text { Gulf of Venice, Gulf of } \\
\text { Trieste }\end{array}$ & 26 Oct -12 Nov 2004 & 5-min & $0.8-3.3$ & $1.6 \pm 0.4$ & Sprovieri \& Pirrone (2008) \\
\hline & Ship $^{\mathrm{G}}$ & Rome - Messina & $\begin{array}{l}26 \text { Oct - } 15 \text { Nov } 2004 \\
15 \text { Jun }-5 \text { Jul } 2005\end{array}$ & $5-\min$ & N/A & $\begin{array}{l}1.6 \\
2.0\end{array}$ & Sprovieri et al. (2010) \\
\hline $\begin{array}{l}\text { Augusta } \\
\text { Basin }\end{array}$ & Ship $^{G}$ & Across the basin & $\begin{array}{l}\text { Nov } 2011 \\
\text { Jul - Jun } 2012\end{array}$ & $5 \mathrm{~s}(20 \mathrm{~km})$ & $\begin{array}{l}0.9-3.1 \\
1.1-3.1\end{array}$ & $\begin{array}{l}1.5 \pm 0.4 \\
2.1 \pm 0.98\end{array}$ & Bagnato et al. (2013) \\
\hline \multirow[t]{4}{*}{ Arctic } & Ground $^{\mathrm{G}}$ & Barrow, Alaska & $1998-2001$ & 5-min & $<0.2-3.7$ & $1.5-2$ & Lindberg et al. (2002) \\
\hline & Ship $^{G}$ & $\begin{array}{l}\text { Götborg - Barrow } \\
\text { Barrow -Chukchi Peninsula } \\
\text { - Wrangel Island - Barrow } \\
\text { Barrow -Longyearbyen } \\
\end{array}$ & Jul - Sep 2005 & $5-\min$ & $1-5.24$ & $\begin{array}{l}1.73 \pm 0.36 \\
\text { Sea ice: } 1.81 \pm 0.41 \\
\text { Sea w/o ice: } 1.55 \pm 0.21\end{array}$ & Sommar et al. (2010) \\
\hline & Ship & Central Arctic & Jul - Sep 2012 & 5-min & $0.15-4.58$ & $1.23 \pm 0.61$ & Yu et al. (2014) \\
\hline & Ground $^{\mathrm{G}}$ & Beaufort Sea & $14-26$ Mar 2009 & 5-min & $0.01-1.51$ & 0.59 & Steffen et al. (2013) \\
\hline \multirow[t]{6}{*}{ Antarctic } & Ground & Ross Island & $1987-1989$ & N/A & $0.02-1.85$ & $0.55 \pm 0.28$ & De More et al. (1993) \\
\hline & Ground & Neumayer $70^{\circ} 39^{\prime} \mathrm{S}, 8^{\circ} 15^{\prime} \mathrm{W}$ & $\begin{array}{l}\text { Jan } 2000-\text { Jan } 2001 \\
\text { Jan-Feb 2000, Dec-Feb } \\
2001 \\
\text { Mar-Jul } 2000 \\
\text { Aug -Nov 2000 }\end{array}$ & $5-\min$ & N/A & $\begin{array}{l}1.063 \pm 0.235 \\
1.043 \pm 0.284 \\
1.146 \pm 0.075 \\
0.968 \pm 0.278 \\
\end{array}$ & Ebinghaus et al. (2002) \\
\hline & Ground $^{\mathrm{G}}$ & Terra Nova Bay, Antarctic & Nov 2000 - Jan 2001 & 5-min & $0.29-2.3$ & $0.9 \pm 0.3$ & Sprovieri et al. (2002) \\
\hline & Ground & Neumayer, Antarctic & Dec $2000-$ Feb 2001 & $5-15-\min$ & $<0.3-2.34$ & $1.08 \pm 0.29$ & Temme et al. (2003b) \\
\hline & Ship $^{G}$ & $\begin{array}{l}65^{\circ} \mathrm{S}-55^{\circ} \mathrm{S} \\
65^{\circ} \mathrm{S}-63^{\circ} \mathrm{S} \text { Antarctica }\end{array}$ & $\begin{array}{l}\text { 14-24 Jan } 2007 \\
25-28 \text { Jan }\end{array}$ & $5-\min$ & N/A & $\begin{array}{l}1.30 \pm 0.16 \\
1.55 \pm 0.38 \\
\end{array}$ & Soerensen et al. (2010) \\
\hline & Ground $^{\mathrm{G}}$ & TRS, Queen Maud Land & Feb 2007 - Jun 2010 & 5-min & $0.02-3.04$ & $0.93 \pm 0.19$ & Pfaffhuber et al. (2012) \\
\hline
\end{tabular}


Table S2. Marine boundary layer GOM ( $\mathrm{pg} \mathrm{m}^{-3}$, units noted otherwise). Units were converted in STP. The units for time resolution are hours (denoted as $\mathrm{h}$ ) and minutes (denoted as min).

\begin{tabular}{|c|c|c|c|c|c|c|c|}
\hline & Platform & Latitude & Sampling Period & Time Resolution & Range & Average $\pm \sigma$ & Reference \\
\hline \multirow[t]{11}{*}{ Atlantic } & Ship ${ }^{\text {DDM }}$ & $35^{\circ} \mathrm{S}-54^{\circ} \mathrm{N}$ & 1977 & 40-min & $0.2-15.3 \mathrm{ng} \mathrm{m}^{-3}$ & N/A & Slemr et al. (1981); \\
\hline & Ship ${ }^{\text {DDM }}$ & $35^{\circ} \mathrm{S}-54^{\circ} \mathrm{N}$ & $1978-1980$ & $20-40-\min$ & $\begin{array}{l}<0.02(<2 \%)(\text { Median }) \\
\text { MMC }:<0.1(<5 \%)\end{array}$ & N/A & Slemr et al. (1985) \\
\hline & Ground & Chesapeake Biol. Lab & $1997-1999$ & $6-24-h$ & N/A & $40 \pm 50$ & Sheu\&Mason (2001) \\
\hline & Ground & $\begin{array}{l}\text { Near Bermuda } \\
\left(31^{\circ} 40^{\prime} \mathrm{N}, 64^{\circ} 10^{\prime} \mathrm{W}\right)\end{array}$ & $\begin{array}{l}\text { Sep \& Dec 1999; Mar } \\
2000\end{array}$ & N/A & $\begin{array}{l}<0.05-6.86 \mathrm{pmol} \mathrm{m}^{-3} \\
(<11-1500)\end{array}$ & $\begin{array}{l}1.38 \pm 1.3 \mathrm{pmol} \mathrm{m}^{-3} \\
(300 \pm 280)\end{array}$ & Mason et al. (2001) \\
\hline & Ground & Florida $\sim 26^{\circ} \mathrm{N}$ & Jun 2000 & 1-h & $<6.9$ & $1.6 \pm 1.5$ & Malcom et al. (2003) \\
\hline & Ship & $22^{\circ} \mathrm{N}-48^{\circ} \mathrm{N}$ & May - Jun 2002 & & $2.8-92.4$ & $13.8 \pm 13.1$ & Laurier et al. (2003) \\
\hline & Ship & $\begin{array}{l}54^{\circ} \mathrm{N}-85^{\circ} \mathrm{N} \\
78^{\circ} \mathrm{N}-85^{\circ} \mathrm{N}\end{array}$ & 16 Jun - 29 Aug 2004 & $\begin{array}{l}\text { 2-h (auto) } \\
2-12 \text {-h (man) }\end{array}$ & $\begin{array}{l}0-22 \\
0-21\end{array}$ & $\begin{array}{l}2.4 \pm 2 \\
2.6 \pm 2.3\end{array}$ & Aspmo et al. (2006) \\
\hline & Ship & $\begin{array}{l}\text { Neumayer - Punta } \\
\text { Arenas }\end{array}$ & Feb 2001 & 1-h & $1-30$ & N/A & Temme et al. (2003b) \\
\hline & Ship & $\begin{array}{l}\text { Norfolk, VA to } \\
\text { Bermuda, to Barbados }\end{array}$ & $\begin{array}{l}15 \text { Aug - 8 Sep } \\
2003\end{array}$ & 2-h & $<3(\mathrm{LOD})-27$ & 5.9 & Laurier et al. (2007) \\
\hline & Ground & $\begin{array}{l}\text { Appledore Island } \\
42.97^{\circ} \mathrm{N}, 70.62^{\circ} \mathrm{W}\end{array}$ & $\begin{array}{l}\text { May } 2007 \text { - Oct } 2007 \\
\text { May } 2007 \text { - Oct } 2010\end{array}$ & 90-min & $\begin{array}{l}0-8.8 \text { ppqv }(0-79) \\
0-11.6 \text { ppqv }(0-104)\end{array}$ & $\begin{array}{l}0.76 \pm 0.88 \text { ppqv }(7 \pm 8) \\
0.4 \pm 0.8 \text { ppqv }(4 \pm 7)\end{array}$ & $\begin{array}{l}\text { Sigler et al. (2009) } \\
\text { Mao \&Talbot (2012) }\end{array}$ \\
\hline & Ship & $\begin{array}{l}58^{\circ} \mathrm{N}-67^{\circ} \mathrm{N} \\
43^{\circ} \mathrm{N}-59^{\circ} \mathrm{N} \\
23^{\circ} \mathrm{N}-45^{\circ} \mathrm{N} \text { Sargasso } \\
\text { Sea } \\
39^{\circ} \mathrm{S}-3^{\circ} \mathrm{S} \text { South Africa }\end{array}$ & $\begin{array}{l}16 \text { Aug - 1Sep } 2006 \\
15-24 \text { Apr } 2007 \\
30 \text { Mar - } 11 \text { Apr } 2007 \\
8-21 \text { Oct } 2006\end{array}$ & 40-min & N/A & $\begin{array}{l}0.4 \pm 3 \\
0.1 \pm 1 \\
1.2 \pm 2 \\
3.4 \pm 424\end{array}$ & $\begin{array}{l}\text { Soerensen et al. } \\
(2010)\end{array}$ \\
\hline \multirow[t]{2}{*}{ Indian } & Ship & $\begin{array}{l}39^{\circ} \mathrm{S}-33^{\circ} \mathrm{S} \\
22^{\circ} \mathrm{S}-17^{\circ} \mathrm{S} \mathrm{W} . \\
\text { Australia } \\
\end{array}$ & $\begin{array}{l}22-29 \text { Oct } 2006 \\
3-6 \text { Nov } 2006\end{array}$ & 40-min & N/A & $\begin{array}{l}4.6 \pm 5 \\
5.0 \pm 6\end{array}$ & $\begin{array}{l}\text { Soerensen et al. } \\
(2010)\end{array}$ \\
\hline & Ground & $\begin{array}{l}\text { Amsterdam Island } \\
37^{\circ} 48^{\prime} \mathrm{S}, 77^{\circ} 34^{\prime} \mathrm{E}\end{array}$ & Jan 2012 to Dec 2013 & 4-h & LOD - 4.07 & 0.34 & Angot et al. (2014) \\
\hline \multirow[t]{5}{*}{ Pacific } & Ship & $\begin{array}{l}\text { Osaka, Japan to } \\
\text { Honolulu, Hawaii }\end{array}$ & $\begin{array}{l}\text { 1 May } 2002-4 \text { Jun } \\
2002\end{array}$ & 2-h & $0.15-9.24$ & 9.5 & Laurier et al. (2003) \\
\hline & Ground & $\begin{array}{l}48.3 \mathrm{~N}, 124.6 \mathrm{~W}, 480 \mathrm{~m} \\
\text { asl }\end{array}$ & May 2001 - May 2002 & 4-h & $\begin{array}{l}\text { Fall 2001 - winter } \\
\text { 2002: <LOD (1.6) } \\
\text { Spring 2002: } 1.6\end{array}$ & N/A & $\begin{array}{l}\text { Weiss-Penzias et al. } \\
\text { (2003) }\end{array}$ \\
\hline & Ground & $\begin{array}{l}26.8^{\circ} \mathrm{N}, 128.2^{\circ} \mathrm{E}, 60 \mathrm{~m} \\
\mathrm{amsl}\end{array}$ & 23 Mar - 2 May 2004 & 3-h & $<20$ & $4.5 \pm 5.4$ & Chand et al. (2008) \\
\hline & Ship & $\begin{array}{l}44^{\circ} \mathrm{S}-26^{\circ} \mathrm{S} \text { E. Australia } \\
27^{\circ} \mathrm{S}-7^{\circ} \mathrm{S} \text { Coral Sea } \\
56^{\circ} \mathrm{S}-26^{\circ} \mathrm{S} \text { New } \\
\text { Zealand } \\
58^{\circ} \mathrm{S}-33^{\circ} \mathrm{S} \text { Chilean } \\
\text { Coast }\end{array}$ & $\begin{array}{l}23 \text { Nov - 15 Dec } 2006 \\
16 \text { Dec } 2006-3 \text { Jan } \\
2007 \\
3 \text { - 14 Jan } 2007 \\
\text { 31 Jan - } 8 \text { Feb } 2007\end{array}$ & 40-min & N/A & $\begin{array}{l}1.9 \pm 3 \\
0.3 \pm 1 \\
0.1 \pm 0 \\
28.6 \pm 30\end{array}$ & $\begin{array}{l}\text { Soerensen et al. } \\
(2010)\end{array}$ \\
\hline & Ground & Mt. Bachelor Obs., & $2005-2011$ & 3-h & $200-700$ & N/A & Timonen et al. (2013) \\
\hline
\end{tabular}




\begin{tabular}{|c|c|c|c|c|c|c|c|}
\hline & & $\begin{array}{l}\text { Oregon, US } 43.981^{\circ} \mathrm{N} \\
121.691^{\circ} \mathrm{W} 2.7 \mathrm{~km} \text { a.s.l. }\end{array}$ & & & & & \\
\hline & Ground & $\begin{array}{l}\text { Puerto Villamil, Isabela } \\
\text { Island } \\
\text { Puerto Baquerizo } \\
\text { Moreno, } \\
\text { San Cristóbal Island }\end{array}$ & $\begin{array}{l}23 \text { Feb-29 Mar } 2011 \\
\text { 10-19 Jun } 2011 \\
10-26 \text { Oct } 2011\end{array}$ & 3-h & $<0.42(\mathrm{LOD})-16$ & $\begin{array}{l}1.1 \pm 1.2 \\
<0.42(\mathrm{LOD}) \\
3.8 \pm 3.4\end{array}$ & Wang et al. (2014) \\
\hline \multirow[t]{2}{*}{ Mediterranean } & Ground & $\begin{array}{l}\text { Palma de Mallorca, } \\
\text { Fuscaldo, Porto Palo, } \\
\text { Anatalya, Neve Yam }\end{array}$ & $\begin{array}{l}13 \text { Nov - 6 Dec } 1998 \\
15 \text { Feb - 1 Mar } 1999 \\
3 \text { May - 17 May } 1999 \\
19 \text { Jul - 2 Aug } 1999\end{array}$ & $2-h$ & N/A & $\begin{array}{l}31.5 \pm 39.2 \\
40.4 \pm 43 \\
52.3 \pm 43.9 \\
32.3 \pm 17.8\end{array}$ & Pirrone et al. (2003) \\
\hline & Ship & $\begin{array}{l}30-40^{\circ} \mathrm{N} \\
\text { Western and eastern } \\
\text { sectors }\end{array}$ & 14 Jul - 9 Aug 2000 & $2-h$ & $0.2-30$ & $8 \pm 8$ & Sprovieri et al. (2003) \\
\hline \multirow[t]{2}{*}{ Adriatic } & Ship & Rome - Messina & 26 Oct -12 Nov 2004 & $2-h$ & $0.1-62.8$ & $6.7 \pm 11.7$ & $\begin{array}{l}\text { Sprovieri \& Pirrone } \\
(2008)\end{array}$ \\
\hline & Ship & Rome - Messina & $\begin{array}{l}26 \text { Oct - 15 Nov } 2004 \\
\text { 15 Jun - 5 Jul } 2005\end{array}$ & 2-h & N/A & $\begin{array}{l}1.6 \\
2.0\end{array}$ & Sprovieri et al. (2010) \\
\hline Dead Sea & Ground & $\begin{array}{l}\text { Ein Bokek, Israel } 31.20^{\circ} \\
\mathrm{N}, 35.37^{\circ} \mathrm{E}\end{array}$ & $\begin{array}{l}29 \text { Jun - 28 Jul } 2009 \\
29 \text { Dec } 2009-17 \text { Jan } \\
2010\end{array}$ & 1-h & $\begin{array}{l}22-450 \text { ppqv } \\
(196-4018)\end{array}$ & N/A & $\begin{array}{l}\text { Obrist et al. (2011); } \\
\text { Moore et al. (2013) }\end{array}$ \\
\hline \multirow[t]{3}{*}{ Arctic } & Ground & Barrow, Alaska, US & $1998-2001$ & 2-h & $0-950$ (at surface) & $\begin{array}{l}5 \mathrm{~m} \text { agl: } 70 \\
100 \text { m agl: } 20 \\
100 \text { m agl: } 2 \\
\end{array}$ & Lindberg et al. (2002) \\
\hline & Ship & $\begin{array}{l}\text { Götborg - Barrow } \\
\text { Barrow -Chukchi } \\
\text { Peninsula - Wrangel } \\
\text { Island - Barrow } \\
\text { Barrow - Longyearbyen }\end{array}$ & Jul - Sep 2005 & $5-h$ & $0.7-10$ & $3.2 \pm 1.7$ & Sommar et al. (2010) \\
\hline & Ground & Beaufort Sea & 14-26 Mar 2009 & 1-h & $3.5-105.4$ & 30.1 & Steffen et al. (2013) \\
\hline \multirow[t]{4}{*}{ Antarctic } & Ground & Ross Island & $1987-1989$ & N/A & $0.00-0.63 \mathrm{DMM}$ & $0.04 \pm 0.08$ & De More et al. (1993) \\
\hline & Ground & Terra Nova Bay & Nov 2000 - Jan 2001 & 2-h & $10.5-334$ & $116.2 \pm 77.8$ & Sprovieri et al. (2002) \\
\hline & Ground & Neumayer & Dec 2000 - Feb 2001 & 1-h & $5-300$ & N/A & Temme et al. (2003b) \\
\hline & Ship & $\begin{array}{l}65^{\circ} \mathrm{S}-55^{\circ} \mathrm{S} \\
65^{\circ} \mathrm{S}-63^{\circ} \mathrm{S} \\
\end{array}$ & $\begin{array}{l}14-24 \text { Jan } 2007 \\
25-28 \text { Jan } 2007\end{array}$ & 40-min & N/A & $\begin{array}{l}0 \pm 0 \\
43 \pm 39\end{array}$ & $\begin{array}{l}\text { Soerensen et al. } \\
(2010)\end{array}$ \\
\hline
\end{tabular}


Table S3. Marine boundary layer PBM ( $\mathrm{pg} \mathrm{m}^{-3}$, noted otherwise). Units were converted in STP. The superscript T stands for total particulate mercury.

\begin{tabular}{|c|c|c|c|c|c|c|c|}
\hline & Platform & Latitude & Sampling Period & Time Resolution & Range & Average $( \pm \sigma)$ & Reference \\
\hline \multirow[t]{9}{*}{ Atlantic } & Ship & $54^{\circ} \mathrm{N}, 10^{\circ} \mathrm{E}-20^{\circ} \mathrm{N}, 67^{\circ} \mathrm{W}$ & Oct - Nov 1973 & $2-4-h$ & $<300$ & N/A & Seiler et al. (1980) \\
\hline & Ship & $15^{\circ} \mathrm{S} 29^{\circ} \mathrm{N}$ & 1980 & N/A & N/A & $0.013 \pm 0.018$ & Slemr et al. (1985) \\
\hline & Ship & $1^{\circ} \mathrm{N}-33^{\circ} \mathrm{N}$ & $1977-1980$ & N/A & N/A & $0.007 \pm 0.004$ & Slemr et al. (1985) \\
\hline & Ship & $\begin{array}{l}4.5^{\circ} \mathrm{N}-4.5^{\circ} \mathrm{S} \text { at } 85^{\circ} \mathrm{W} ; \\
155^{\circ} \mathrm{W}-93^{\circ} \mathrm{W} \text { in the } \\
\text { equator }\end{array}$ & $\begin{array}{l}16 \text { May - } 20 \text { June } \\
1996\end{array}$ & 24-h & $\begin{array}{l}5-25 \text { fmol m}{ }^{-3} \\
(1-5.5)\end{array}$ & $\begin{array}{l}12 \pm 1 \mathrm{fmol} \mathrm{m}^{-3} \\
(2.6 \pm 0.2)\end{array}$ & Lamborg et al. (1999) \\
\hline & Ground & Chesapeake Biol. Lab & $1997-1999$ & $6-24-h$ & N/A & $0.02 \pm 0.05$ & Sheu\&Mason (2001) \\
\hline & Ship & $\begin{array}{l}\text { Near Bermuda } \\
\left(31^{\circ} 40^{\prime} \mathrm{N}, 64^{\circ} 10^{\prime} \mathrm{W}\right)\end{array}$ & $\begin{array}{l}\text { Sep \& Dec 1999; Mar } \\
2000\end{array}$ & N/A & N/A & $<0.01 \mathrm{pmol} \mathrm{m}^{-3}$ & Mason et al. (2001) \\
\hline & Ground & Florida $\sim 26^{\circ} \mathrm{N}$ & & 4-h & $<11$ & $6.3 \pm 4.4$ & Malcom et al. (2003) \\
\hline & Ship & $78^{\circ} \mathrm{N}-85^{\circ} \mathrm{N}$ & 16 Jun - 29 Aug 2004 & $2-12-h$ & $0.2-6.3$ & $2.4 \pm 1.1$ & Aspmo et al. (2006) \\
\hline & Ground & $\begin{array}{l}\text { Appledore Island } \\
42.97^{\circ} \mathrm{N}, 70.62^{\circ} \mathrm{W}\end{array}$ & Apr 2009 - Aug 2010 & 90-min & $\begin{array}{l}0-39 \text { ppqv } \\
(0-348)\end{array}$ & $\begin{array}{l}0.34 \pm 0.78 \mathrm{ppqv} \\
(3 \pm 7)\end{array}$ & Mao \& Talbot (2012) \\
\hline Indian & Ground & $\begin{array}{l}\text { Amsterdam Island } \\
37^{\circ} 48^{\prime} \mathrm{S}, 77^{\circ} 34^{\prime} \mathrm{E}\end{array}$ & Jan 2012 to Dec 2013 & 4-h & $<\mathrm{DL}-12.67$ & 0.67 & Angot et al. (2014) \\
\hline \multirow[t]{4}{*}{ Pacific } & Ground & $\begin{array}{l}48.3 \mathrm{~N}, 124.6 \mathrm{~W}, 480 \mathrm{~m} \\
\text { asl }\end{array}$ & May 2001 - May 2002 & 24-h & N/A & Spring 2002: 0.5 & $\begin{array}{l}\text { Weiss-Penzias et al. } \\
(2003)\end{array}$ \\
\hline & Ground & $\begin{array}{l}26.8^{\circ} \mathrm{N}, 128.2^{\circ} \mathrm{E}, \\
60 \mathrm{~m} \text { amsl }\end{array}$ & 23 Mar - 2 May 2004 & 3-h & $<17$ & $3.0 \pm 2.5$ & Chand et al. (2008) \\
\hline & Ground & $\begin{array}{l}\text { Mt. Bachelor Obs., } \\
\text { Oregon, US } 43.981^{\circ} \mathrm{N} \\
121.691^{\circ} \mathrm{W} 2.7 \mathrm{~km} \text { a.s.l. }\end{array}$ & $2005-2011$ & 3-h & $\begin{array}{l}\text { MBL events } \\
<12\end{array}$ & & Timonen et al. (2013) \\
\hline & Ground & $\begin{array}{l}\text { Puerto Villamil, Isabela } \\
\text { Island } 0^{\circ} 57^{\prime} \mathrm{S}, 90^{\circ} 58^{\prime} \mathrm{W} \\
\text { Puerto Baquerizo } \\
\text { Moreno, San Cristóbal } \\
\text { Island, } 0^{\circ} 54^{\prime} \mathrm{S}, 89^{\circ} 36^{\prime} \mathrm{W}\end{array}$ & $\begin{array}{l}23 \text { Feb-29 Mar } 2011 \\
\text { 10-19 Jun } 2011 \\
\text { 10-26 Oct } 2011\end{array}$ & 3-h & N/A & $\begin{array}{l}0.6 \pm 0.7 \\
<\mathrm{LOD} \\
1.1 \pm 1.1\end{array}$ & Wang et al. (2014) \\
\hline Adriatic & Ship & $\begin{array}{l}\text { Gulf of Venice, Gulf of } \\
\text { Trieste }\end{array}$ & 26 Oct -12 Nov 2004 & $2-\mathrm{h}$ & $0.04-51$ & $4.5 \pm 8$ & $\begin{array}{l}\text { Sprovieri \& Pirrone } \\
(2008)\end{array}$ \\
\hline Baltic Sea & & $\begin{array}{l}\text { Gdynia } 54^{\circ} 31^{\prime} \mathrm{N}, 18^{\circ} \\
48^{\prime} \mathrm{E}\end{array}$ & $\begin{array}{l}18 \text { Dec } 2007-15 \text { Dec } \\
2008\end{array}$ & 24-h & $2-142$ & $\begin{array}{l}20 \pm 18 \\
24 \pm 22(\text { cold }) \\
15 \pm 7(\text { warm }) \\
\end{array}$ & $\begin{array}{l}\text { Beldowska et al. } \\
\text { (2012) }\end{array}$ \\
\hline \multirow[t]{2}{*}{ Arctic } & Ship & $\begin{array}{l}\text { Götborg - Barrow } \\
\text { Barrow -Chukchi } \\
\text { Peninsula - Wrangel } \\
\text { Island - Barrow } \\
\text { Barrow - Longyearbyen }\end{array}$ & Jul - Sep 2005 & $5-\mathrm{h}$ & $0.2-3.2$ & $1 \pm 0.7$ & Sommar et al. (2010) \\
\hline & Ground & Beaufort Sea & $14-26$ Mar 2009 & 1-h & $47.1-900.1$ & 393.5 & Steffen et al. (2013) \\
\hline Antarctic & Ground $^{\mathrm{T}}$ & $\begin{array}{l}\text { Neumayer } 70^{\circ} 39^{\prime} \mathrm{S}, \\
8^{\circ} 15^{\prime} \mathrm{W}\end{array}$ & Dec 2000 - Feb 2001 & 48-h & $15-120$ & $\sim 38$ & Temme et al. (2003) \\
\hline
\end{tabular}


Table S4: Continental boundary layer TGM or GEM $\left(\mathrm{ng} \mathrm{m}^{-3}\right)$ at remote, rural, urban, and high elevation sites

\begin{tabular}{|c|c|c|c|c|c|}
\hline Location (elevation in m.a.s.l.) & Sampling period & $\begin{array}{l}\text { Time } \\
\text { resolution }\end{array}$ & Range & Mean $( \pm \sigma)$ & Reference \\
\hline \multicolumn{6}{|l|}{ Remote } \\
\hline $\begin{array}{l}\text { Experimental Lakes Area, Ontario, Canada, } \\
49^{\circ} 39^{\prime} 50^{\prime \prime} \mathrm{N}, 93^{\circ} 43^{\prime} 16^{\prime \prime} \mathrm{W}(369 \mathrm{~m})\end{array}$ & May 2005 - Dec 2006 & $1-\mathrm{h}$ & $0.75-3.82$ & $1.56 \pm 0.22$ & Cheng et al. (2012) \\
\hline Salmon Falls Creek Reservoir, Idaho, USA (1510m) & $\begin{array}{l}2 \text { weeks per season during summer } \\
2005 \text {-fall 2006; all of summer } 2006\end{array}$ & 5 -min & & $1.3-1.9$ & Abbott et al. (2008) \\
\hline $\begin{array}{l}\text { Devil's Lake State Park, Wisconsin, USA, } 43^{\circ} 25^{\prime} 48^{\prime \prime N} \text {, } \\
89^{\circ} 40^{\prime} 48^{\prime \prime} \mathrm{W}(389 \mathrm{~m})\end{array}$ & Apr 2003 - Apr 2004 & 5 -min & $0.1-9.1$ & $1.6 \pm 0.3$ & Manolopoulos et al. (2007) \\
\hline $\begin{array}{l}\text { Devil's Lake State Park, Wisconsin, USA, } 43^{\circ} 26^{\prime} 5^{\prime \prime N} \text {, } \\
89^{\circ} 40^{\prime} 48^{\prime \prime} \mathrm{W}\end{array}$ & Apr 2003 - Mar 2004 & 5 -min & & $1.61 \pm 0.01$ & Rutter et al. (2008) \\
\hline $\begin{array}{l}\text { Mt. Horeb, Wisconsin, USA, } 42^{\circ} 57^{\prime} 36^{\prime \prime} \mathrm{N}, 89^{\circ} 42^{\prime} \mathrm{W} \\
(331 \mathrm{~m})\end{array}$ & June \& Sep 2004 & $5-\min$ & & $1.34 \pm 0.26$ & Manolopoulos et al. (2007) \\
\hline Contiguous USA & Jan 2009 - Dec 2011 & 1-h & $0.2-11.3$ & $1.31 \pm 0.29$ & Gay et al. (2013) \\
\hline \multicolumn{6}{|l|}{ Rural } \\
\hline $\begin{array}{l}\text { Multiple sites across Canada, } 42^{\circ} 10^{\prime}-82^{\circ} 30^{\prime} \mathrm{N}, 135^{\circ} 37^{\prime}- \\
62^{\circ} 19^{\prime} \mathrm{W}\end{array}$ & $1994-2011$ & $5-\min$ & & $1.21-1.93$ & Cole et al. (2014) \\
\hline Maryhill, Ontario, Canada, $43^{\circ} 31^{\prime} \mathrm{N}, 80^{\circ} 25^{\prime} \mathrm{W}$ & Oct 2004 - Nov 2004 & $5-\min$ & & $1.8 \pm 0.2$ & Cobbett and Van Heyst (2007) \\
\hline Central Alberta, Canada & $2004-2007$ & 5 -min & $0.7-9.5$ & $1.5-1.6$ & Mazur et al. (2009) \\
\hline $\begin{array}{l}\text { Fort McMurray, Alberta, Canada, } 56^{\circ} 45^{\prime} \mathrm{N}, \\
111^{\circ} 28^{\prime} 48^{\prime \prime} \mathrm{W}(362 \mathrm{~m})\end{array}$ & Oct 2010 - May 2013 & 5 -min & $0.64-4.43$ & $1.45 \pm 0.18$ & Parsons et al. (2013) \\
\hline $\begin{array}{l}\text { Huntington Forest, New York, USA, } 43^{\circ} 58^{\prime} 12^{\prime \prime N} \text {, } \\
74^{\circ} 13^{\prime} 12^{\prime \prime} \mathrm{W}(500 \mathrm{~m})\end{array}$ & Jun 2006 - May 2007 & $1-\mathrm{h}$ & $0.5-2.5$ & $1.4 \pm 0.4$ & Choi et al. (2008) \\
\hline $\begin{array}{l}\text { Huntington Forest, New York, USA,, } 43^{\circ} 58^{\prime} 12^{\prime \prime} \mathrm{N}, \\
74^{\circ} 13^{\prime} 12^{\prime \prime} \mathrm{W}(510 \mathrm{~m})\end{array}$ & Dec 2007 - Nov 2009 & $2-\mathrm{h}$ & $0.2-3.7$ & $1.3 \pm 0.4$ & Choi et al. (2013) \\
\hline $\begin{array}{l}\text { Huntington Forest, New York, USA, } 43^{\circ} 58^{\prime} 12^{\prime \prime} \mathrm{N}, \\
74^{\circ} 13^{\prime} 12^{\prime \prime} \mathrm{W}(500 \mathrm{~m})\end{array}$ & Jan 2009 - Dec 2010 & $2 \mathrm{~h}$ & $0.2-3.4$ & & Cheng et al. (2013) \\
\hline Atlanta, Georgia, USA & $2005-2008$ & 5 -min & & 1.35 & Nair et al. (2012) \\
\hline Wisconsin, USA, $43^{\circ} 26^{\prime} 24^{\prime \prime} \mathrm{N}, 89^{\circ} 40^{\prime} 48^{\prime \prime} \mathrm{W}(389 \mathrm{~m})$ & 2003-2009 & 5 -min & $0.45-6.48$ & $1.49-1.82$ & Engle et al. (2010) \\
\hline North Dakota, USA, $48^{\circ} 38^{\prime} 60^{\prime \prime} \mathrm{N}, 102^{\circ} 24^{\prime} \mathrm{W}(692 \mathrm{~m})$ & 2003-2009 & 5 -min & $0.99-5.13$ & $1.61 \pm 0.22$ & Engle et al. (2010) \\
\hline Virginia, USA, 38³1'12"N, 78²6'24"W (1074m) & 2003-2009 & $5-\min$ & $<\mathrm{dl}-3.63$ & $1.27 \pm 0.31$ & Engle et al. (2010) \\
\hline Dexter, Michigan, USA, $42^{\circ} 25^{\prime} 12^{\prime \prime} \mathrm{N}, 83^{\circ} 54^{\prime} \mathrm{W}$ & 2004 & $1-\mathrm{h}$ & $\begin{array}{ll}0.26- \\
14.82 \\
\end{array}$ & $1.6 \pm 0.6$ & Liu et al. (2010) \\
\hline Holland, Illinois, USA, $42^{\circ} 46^{\prime} 4.8^{\prime \prime} \mathrm{N}, 86^{\circ} 8^{\prime} 56.4^{\prime \prime W}$ & 1 Jul 2007 - 8 Nov 2007 & 1-h & $\leq 6.0$ & $1.3 \pm 0.3$ & Gratz et al. (2013) \\
\hline $\begin{array}{l}\text { San Francisco Bay Area, California, USA } \\
37^{\circ} 11^{\prime} 24^{\prime \prime N}, 121^{\circ} 46^{\prime} 12^{\prime \prime} \mathrm{W}(149 \mathrm{~m})\end{array}$ & $\begin{array}{l}\text { 9 Sep 2005-27 Sep 2005, } 21 \text { Oct } \\
\text { 2008-30 Oct } 2008\end{array}$ & 5-min & $0.84-11.7$ & $1.85-2.37$ & Rothenberg et al. (2010) \\
\hline
\end{tabular}




\begin{tabular}{|c|c|c|c|c|c|}
\hline Central Wisconsin, USA & Jun 2007 - Nov 2007 & $5-\min$ & & $1.3-1.4$ & Kolker et al. (2010) \\
\hline Athens, Ohio, USA $39^{\circ} 18^{\prime} \mathrm{N}, 82^{\circ} 7^{\prime} \mathrm{W}(330 \mathrm{~m})$ & Jul 2004 - Jul 2005 & 1-h & $0.78-4.38$ & $1.62 \pm 0.24$ & Yatavelli et al. (2006) \\
\hline Mexico, $21^{\circ} 8^{\prime} \mathrm{N}, 98^{\circ} 25^{\prime} \mathrm{W}$ & 3 days in Oct 2002 & $5-\min$ & $1.13-2.93$ & $1.32 \pm 0.33$ & De la Rosa et al. 2004 \\
\hline 13 UK sites & $2004-2013$ & $1-4$ weeks & & $1.67 \pm 0.07$ & Brown et al. (2015) \\
\hline Lichwin, Poland, $49^{\circ} 52.957^{\prime} \mathrm{N}, 20^{\circ} 57.024^{\prime} \mathrm{E}$ & 19 Aug $2003-29$ Aug 2003 & 23-h & & $1.63 \pm 0.35$ & Zielonka et al. (2005) \\
\hline Lichwin, Poland, $49^{\circ} 52.957^{\prime} \mathrm{N}, 20^{\circ} 57.024^{\prime} \mathrm{E}$ & 6 Jan 2004 - 3 Feb 2004 & 23-h & $\leq 7.02$ & $4.15 \pm 1.33$ & Zielonka et al. (2005) \\
\hline Chongqing, China & Aug 2006 - Sep 2007 & 30-min & & $6.14 \pm 1.3$ & Yang et al. (2009) \\
\hline Chongqing, China & Aug 2006 - Sep 2007 & 30-min & & $4.32 \pm 1.04$ & Yang et al. (2009) \\
\hline $\begin{array}{l}\text { Wanqingsha, Pearl River Delta, China, } \\
22^{\circ} 42^{\prime} \mathrm{N}, 113^{\circ} 33^{\prime} \mathrm{E}\end{array}$ & Nov 2008 & $5-\min$ & $0.5-18.67$ & $2.94 \pm 2.02$ & Li et al. (2011) \\
\hline Beijing, China, $40^{\circ} 28^{\prime} 51^{\prime \prime N}, 116^{\circ} 46^{\prime} 30^{\prime E}$ (220m) & Dec 2008 - Nov 2009 & 5 -min & $\begin{array}{l}0.39- \\
14.83\end{array}$ & $3.22 \pm 1.74$ & Zhang et al. (2013) \\
\hline 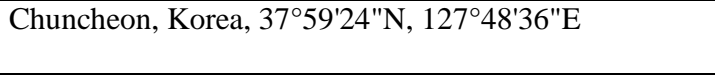 & $2006-2008$ & 24-h & $\begin{array}{l}0.26- \\
10.75 \\
\end{array}$ & $2.12 \pm 1.47$ & Han et al. (2014) \\
\hline $\begin{array}{l}\text { Dinghu, Pearl River Delta, China, } 23^{\circ} 9^{\prime} 51^{\prime N}, \\
112^{\circ} 32^{\prime} 57^{\prime \prime E}\end{array}$ & Oct 2009 - Apr 2010 & 5 -min & $1.87-29.9$ & $5.07 \pm 2.89$ & Chen et al. (2013) \\
\hline \multicolumn{6}{|l|}{ Urban } \\
\hline $\begin{array}{l}\text { Halifax, Nova Scotia, Canada, 44³9'54.71"N, } \\
63^{\circ} 34^{\prime} 9.69^{\prime \prime} \mathrm{W}(125 \mathrm{~m})\end{array}$ & Jan 2010 - Dec 2011 & 1-h & & $1.7 \pm 1.0$ & Cheng et al. (2014) \\
\hline Windsor, Ontario, Canada, $42^{\circ} 18.27^{\prime} \mathrm{N}, 83^{\circ} 3.98^{\prime} \mathrm{W}$ & 2007 & 1-h & $0.83-40.9$ & $2.0 \pm 1.6$ & Xu and Akhtar (2010) \\
\hline Toronto, Ontario, Canada, $43^{\circ} 40^{\prime} \mathrm{N}, 79^{\circ} 24^{\prime} \mathrm{W}$ & Dec 2003 - Nov 2004 & 5 -min & $0.5-44.1$ & $4.5 \pm 3.1$ & Song et al. (2009) \\
\hline Windsor, Ontario, Canada, $42^{\circ} 18.27^{\prime} \mathrm{N}, 83^{\circ} 3.98^{\prime} \mathrm{W}$ & $2007-2011$ & 1-h & $0.3-57$ & $2.0 \pm 1.3$ & Xu et al. (2014) \\
\hline Birmingham, Alabama, USA & $2005-2008$ & $5-\min$ & & 2.12 & Nair et al. (2012) \\
\hline Houston, Texas, USA, $29^{\circ} 42^{\prime} 36^{\prime \prime N}, 9^{\circ} 20^{\prime} 24^{\prime \prime W}(75 \mathrm{~m})$ & Aug 2011 - Oct 2012 & $2.5-\min$ & $\begin{array}{l}0.72- \\
43.54\end{array}$ & $1.6 \pm 0.6$ & Lan et al. (2014) \\
\hline Reno, Nevada, USA, 39³4'N, 11948'W (1509m) & $2002-2005$ & $2.5-\min$ & $0.9-8.6$ & 2.3 & Stamenkovich et al. (2007) \\
\hline Reno, Nevada, USA, 39³4'12"N, $119^{\circ} 47^{\prime} \mathrm{W}$ (1509m) & Nov 2004 - Nov 2007 & $2 \mathrm{~h}$ & $0.5-6.4$ & $1.6 \pm 0.5$ & Peterson et al. (2009) \\
\hline $\begin{array}{l}\text { Desert Research Institute, Nevada, USA, } \\
39^{\circ} 34^{\prime} 12^{\prime \prime} \mathrm{N}, 119^{\circ} 47^{\prime} \mathrm{W}(1497 \mathrm{~m})\end{array}$ & Jun 2007 - Aug 2007 & $2-\mathrm{h}$ & $0.6-2.5$ & $1.2 \pm 0.3$ & Weiss-Penzias et al. (2009) \\
\hline $\begin{array}{l}\text { NV98, Nevada, USA, } 39^{\circ} 30^{\prime} 36^{\prime \prime} \mathrm{N}, 119^{\circ} 43^{\prime} 12^{\prime \prime} \mathrm{W} \\
(1340 \mathrm{~m})\end{array}$ & Jun 2007 - Aug 2007 & 2-h & $0.9-4.6$ & $1.7 \pm 0.6$ & Weiss-Penzias et al. (2009) \\
\hline Illinois, USA, 38³6'36"N, 909'36"W (206m) & $2003-2009$ & 5 -min & $0.05-324$ & $2.09-2.54$ & Engle et al. (2010) \\
\hline Wisconsin, USA, $43^{\circ} 4^{\prime} 48^{\prime \prime} \mathrm{N}, 8^{\circ} 52^{\prime} 48^{\prime \prime} \mathrm{W}(184 \mathrm{~m})$ & $2003-2009$ & 5 -min & $1.29-24.7$ & $2.26-2.94$ & Engle et al. (2010) \\
\hline Detroit, Michigan, USA, $42^{\circ} 10^{\prime} 48^{\prime \prime} \mathrm{N}, 83^{\circ} 3^{\prime} 36^{\prime \prime W}$ & 2004 & 1-h & $0.36-25.6$ & $2.5 \pm 1.4$ & Liu et al. (2010) \\
\hline
\end{tabular}




\begin{tabular}{|c|c|c|c|c|c|}
\hline $\begin{array}{l}\text { San Francisco Bay Area, California, USA, } 37^{\circ} 25^{\prime} 12^{\prime \prime N} \text {, } \\
122^{\circ} 22^{\prime} 24^{\prime \prime}(11 \mathrm{~m})\end{array}$ & $\begin{array}{l}30 \text { Aug } 2009 \text { - } 9 \text { Sep 2005, } 19 \text { Sep } \\
2008 \text { - } 30 \text { Sep } 2008\end{array}$ & 5 -min & $0.53-8.19$ & $1.74-1.76$ & Rothenberg et al. (2010) \\
\hline $\begin{array}{l}\text { Rochester, New York, USA, } 43^{\circ} 8^{\prime} 46 " \mathrm{~N}, 7^{\circ} 32^{\prime} 54^{\prime \prime W} \\
(137 \mathrm{~m})\end{array}$ & $\begin{array}{l}\text { Dec } 2007 \text { - Apr 2008; Dec } 2008- \\
\text { Apr } 2009\end{array}$ & 5-min & & 1.6 & Wang et al. (2013) \\
\hline 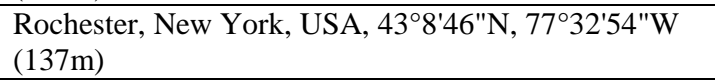 & $\begin{array}{l}\text { Dec } 2007 \text { - Apr } 2008 \text { coal power } \\
\text { plant in operation }\end{array}$ & 5 -min & & 1.8 & Wang et al. (2013) \\
\hline 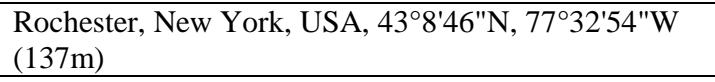 & $\begin{array}{l}\text { Dec } 2007 \text { - Apr } 2009 \text { coal power } \\
\text { plant shutdown }\end{array}$ & 5 -min & & 1.5 & Wang et al. (2013) \\
\hline 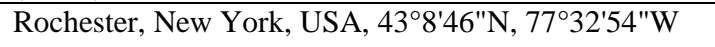 & Sep 2008 - Jun 2013 & $2-h$ & $1.17-1.61$ & $1.40 \pm 0.26$ & Civerolo et al. (2014) \\
\hline New York City, USA, $40^{\circ} 52^{\prime} 05^{\prime \prime} \mathrm{N}, 73^{\circ} 52^{\prime} 42^{\prime \prime} \mathrm{W}$ & Sep 2008 - Jun 2013 & 2-h & $1.20-1.92$ & $1.52 \pm 0.36$ & Civerolo et al. (2014) \\
\hline Milwaukee, Wisconsin, USA, $43^{\circ} 6^{\prime} 29^{\prime \prime N}, 87^{\circ} 53^{\prime} 2^{\prime \prime W}$ & Jun 2004 - May 2005 & 5-min & & $2.48 \pm 0.02$ & Rutter et al. (2008) \\
\hline $\begin{array}{l}\text { Rochester, New York, USA, } 43^{\circ} 8^{\prime} 46^{\prime \prime} \mathrm{N}, 77^{\circ} 32^{\prime} 54^{\prime \prime W} \\
(136 \mathrm{~m})\end{array}$ & Dec 2007 - Nov 2009 & $2-h$ & $0.9-6.4$ & $1.6 \pm 0.4$ & Choi et al. (2013) \\
\hline Chicago, Illinois, USA, $41^{\circ} 47^{\prime} 27.6^{\prime \prime N}, 87^{\circ} 36^{\prime} 7.2^{\prime \prime W}$ & 1 Jul 2007 - 8 Nov 2007 & 1-h & $\leq 16.5$ & $2.5 \pm 1.5$ & Gratz et al. (2013) \\
\hline $\begin{array}{l}\text { Oxford, Mississippi, USA, } 34^{\circ} 21^{\prime} 50^{\prime \prime} \mathrm{N}, 89^{\circ} 32^{\prime} 60^{\prime \prime} \mathrm{W} \\
(150 \mathrm{~m})\end{array}$ & Jul 2011 - Jun 2012 & 5 -min & \begin{tabular}{|l|}
$0.8-7.9$ \\
\end{tabular} & $1.54 \pm 0.32$ & Jiang et al. (2013) \\
\hline Contiguous U.S. & Jan 2009 - Dec 2011 & 1-h & $0.4-237.7$ & $1.65 \pm 1.36$ & Gay et al. (2013) \\
\hline Mexico City, $19^{\circ} 29^{\prime} 21.4^{\prime \prime} \mathrm{N}, 9^{\circ} 8^{\prime} 51.8^{\prime \prime} \mathrm{W}$ & Mar 2006 & 5 -min & & $7.2 \pm 4.8$ & Rutter et al. (2009) \\
\hline Mexico, $22^{\circ} 44^{\prime} \mathrm{N}, 102^{\circ} 28^{\prime} \mathrm{W}$ & 3 days in Sep 2002 & 5 -min & \begin{tabular}{|l|}
$0.26-$ \\
702.32
\end{tabular} & $71.82 \pm 82.03$ & De la Rosa et al. (2004) \\
\hline Mexico City, $19^{\circ} 21^{\prime} \mathrm{N} ; 99^{\circ} 4^{\prime} \mathrm{W}$ & 3 days in Oct 2002 & $5-\min$ & $2.8-34.21$ & $9.81 \pm 3.97$ & De la Rosa et al. (2004) \\
\hline São Paulo State, Brazil & $2002-2003$ & 2-h & & $7.0 \pm 5.8$ & Fostier and Michelazzo, (2006) \\
\hline Oxford, UK & Feb 2008 - Apr 2008 & 5 -min & $1.32-23.2$ & $3.17 \pm 1.59$ & Witt et al. (2010) \\
\hline UK sites & $2004-2013$ & $1-4$ weeks & & $2.07 \pm 0.03$ & Brown et al. (2015) \\
\hline UK sites & $2004-2013$ & 1-4 weeks & & $2.01 \pm 0.12$ & Brown et al. (2015) \\
\hline UK sites & $2003-2013$ & $1-4$ weeks & & $3.74 \pm 0.7$ & Brown et al. (2015) \\
\hline Göteborg, Sweden, $57^{\circ} 42^{\prime} \mathrm{N}, 11^{\circ} 54^{\prime} \mathrm{E}$ & Feb 2005 & 1-h & $1.35-6.42$ & $1.96 \pm 0.38$ & Li et al. (2008) \\
\hline Seoul, Korea, $37^{\circ} 30^{\prime} 50.4^{\prime \prime N}, 127^{\circ} 0 ' 3.6^{\prime \prime} \mathrm{E}$ & Feb 2005 - Dec 2006 & 5 -min & & $3.44 \pm 2.13$ & Choi et al. (2009) \\
\hline Seoul, Korea, $37^{\circ} 30^{\prime} 50.4^{\prime \prime} \mathrm{N}, 127^{\circ} 0^{\prime} 3.6^{\prime \prime} \mathrm{E}$ & $2006-2008$ & 24-h & \begin{tabular}{|l|}
$0.19-$ \\
149.84
\end{tabular} & $3.72 \pm 2.96$ & Han et al. (2014) \\
\hline $\begin{array}{l}\text { Guro-gu, Nowon-gu, Songpa-gu, and Yongsan-gu, } \\
\text { Seoul, Korea, } 27^{\circ} 31^{\prime}-37^{\circ} 39^{\prime} \mathrm{N}, 127^{\circ} 7^{\prime}-126^{\circ} 53^{\prime} \mathrm{W}\end{array}$ & $2010-2011$ & 1-h & & $3.28-3.47$ & Kim et al. (2013) \\
\hline Fukuoka, Japan, 3332'55"N, 130²1'52"E & Jun 2012 - May 2013 & $5-\min$ & $1.47-6.39$ & $2.33 \pm 0.49$ & Marumoto et al. (2015) \\
\hline Chongqing, China & Aug 2006 - Sep 2007 & 30-min & & $7.07 \pm 1.04$ & Yang et al. (2009) \\
\hline
\end{tabular}




\begin{tabular}{|c|c|c|c|c|c|}
\hline $\begin{array}{l}\text { Guangzhou, Pearl River Delta, China, 237'26.8"N, } \\
113^{\circ} 21^{\prime} 17.4^{\prime \prime E}\end{array}$ & Nov 2010 - Oct 2011 & 5 -min & $2.66-11.1$ & $4.60 \pm 1.36$ & Chen et al. (2013) \\
\hline Nanjing, China, $32^{\circ} 2^{\prime} \mathrm{N}, 118^{\circ} 46^{\prime} 48^{\prime \prime E}$ & Jan 2011 - Dec 2011 & $5-\min$ & $0.8-180$ & $7.9 \pm 7.0$ & Zhu et al. (2012) \\
\hline \multicolumn{6}{|l|}{ High elevation } \\
\hline $\begin{array}{l}\text { Pad Monadnock, New Hampshire, USA, 4251'36"N, } \\
71^{\circ} 52^{\prime} 48^{\prime \prime} W(700 \mathrm{~m})\end{array}$ & Jun 2005 - May 2007 & 5 -min & $1.08-1.69$ & $1.26-1.54$ & Mao et al. (2008) \\
\hline $\begin{array}{l}\text { Pad Monadnock, New Hampshire, USA, } 42^{\circ} 51^{\prime} 36^{\prime \prime N}, \\
71^{\circ} 52^{\prime} 48^{\prime \prime} \mathrm{W}(700 \mathrm{~m})\end{array}$ & 2007 & 5 -min & $0.88-2.58$ & $1.40 \pm 0.19$ & Sigler et al. (2009) \\
\hline $\begin{array}{l}\text { Look Rock, Tennessee, } 35^{\circ} 36^{\prime} 36^{\prime \prime N}, 83^{\circ} 54^{\prime} 36^{\prime \prime W} \\
(813 \mathrm{~m})\end{array}$ & Spring-Summer 2004 & $1-\mathrm{h}$ & & 1.65 & Valente et al. (2007) \\
\hline Storm Peak Laboratory, Colorado, USA (3200m) & Oct 2006 - May 2007 & 1-h & $1.06-2.15$ & $1.51 \pm 0.12$ & Obrist et al. (2008) \\
\hline Storm Peak Laboratory, Colorado, USA (3220m) & Apr 2008 - Jul 2008 & $1-\mathrm{h}$ & $1.2-5.0$ & $1.6 \pm 0.3$ & Faïn et al. (2009) \\
\hline $\begin{array}{l}\text { Mt. Bachelor Observatory, Oregon, USA, } 43^{\circ} 58^{\prime} 48^{\prime \prime N} \text {, } \\
121^{\circ} 41^{\prime} 24^{\prime \prime} \mathrm{W}(2700 \mathrm{~m})\end{array}$ & May 2005 - Aug 2005 & 3-h & $1.36-1.83$ & $1.54 \pm 0.18$ & Swartzendruber et al. (2006) \\
\hline 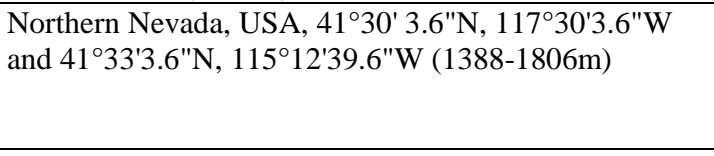 & $\begin{array}{l}29 \text { Mar } 2005 \text { - } 4 \text { Apr 2005, } 26 \text { Sep } \\
2005 \text { - } 8 \text { Aug 2005, 22 Oct } 2005- \\
28 \text { Oct 2005, 24 Jan } 2006-30 \\
\text { Jan2006 }\end{array}$ & 5 -min & $\leq 106$ & $2.5-3.0$ & Lyman and Gustin (2008) \\
\hline Northern Nevada, USA, $41^{\circ} 30^{\prime} \mathrm{N}, 117^{\circ} 30^{\prime} \mathrm{W}(1388 \mathrm{~m})$ & Jun 2007 - Aug 2007 & $2-\mathrm{h}$ & $0.6-18.1$ & $1.8 \pm 1.4$ & Weiss-Penzias et al. (2009) \\
\hline $\begin{array}{l}\text { Contiguous U.S. high elevation sites and Mt. Lulin, } \\
\text { Taiwan }\end{array}$ & Jan 2009 - Dec 2011 & 1-h & $0.4-8.3$ & $1.45 \pm 0.51$ & Gay et al. (2013) \\
\hline $\begin{array}{l}\text { Mt. Gongga, China, } 29^{\circ} 20^{\prime}-30^{\circ} 20^{\prime} \mathrm{N}, 101^{\circ} 30^{\prime}- \\
102^{\circ} 15^{\prime} \mathrm{E}(1640 \mathrm{~m})\end{array}$ & May 2005 - Jun 2006 & $5-15 \min$ & $\begin{array}{l}0.52- \\
21.03 \\
\end{array}$ & $3.98 \pm 1.62$ & Fu et al. (2008) \\
\hline $\begin{array}{l}\text { Mt. Gongga, China, } 29^{\circ} 20^{\prime}-30^{\circ} 20^{\prime} \mathrm{N}, 101^{\circ} 30^{\prime}- \\
102^{\circ} 15^{\prime} \mathrm{E}(1640 \mathrm{~m})\end{array}$ & Jun 2006 - Jun 2007 & 5 -min & $0.9-43.7$ & $3.91 \pm 1.2$ & Fu et al. (2009) \\
\hline Mt. Leigong, China, $23^{\circ} 23^{\prime} 24^{\prime \prime N}, 108^{\circ} 12^{\prime} \mathrm{E}(2178 \mathrm{~m})$ & May 2008 - May 2009 & 10-min & $0.41-23.9$ & $2.80 \pm 1.51$ & Fu et al. (2010a) \\
\hline Waliguan, China, 36²17'12"N, 10053'52.7"E, (3816m) & Sep 2007 - Sep 2008 & 10-min & & $1.98 \pm 0.98$ & Fu et al. (2012a) \\
\hline Mt. Changbai, China, $42^{\circ} 24^{\prime} \mathrm{N}, 128^{\circ} 28^{\prime} \mathrm{E}(738.1 \mathrm{~m})$ & Aug 2005 - Jul 2006 & 1-h & $1.28-9.49$ & $3.58 \pm 1.78$ & Wan et al. (2009a) \\
\hline Mt. Changbai, China, $42^{\circ} 24^{\prime} 0.9^{\prime \prime N}, 128^{\circ} 6^{\prime} 45^{\prime \prime E ~(741 m) ~}$ & Oct 2008 - Oct 2010 & 5 -min & $<\mathrm{dl}-9.5$ & $1.60 \pm 0.51$ & Fu et al. (2012b) \\
\hline 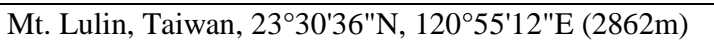 & Apr 2006 - Sep 2007 & 5 -min & $0.24-5.75$ & $1.73 \pm 0.61$ & Sheu et al. (2010) \\
\hline $\begin{array}{l}\text { Shangri-La, Tibetan Plateau, China, } 28^{\circ} 11^{\prime} \mathrm{N}, 99^{\circ} 44^{\prime} \mathrm{E} \\
(3580 \mathrm{~m})\end{array}$ & Nov 2009 - Nov 2010 & 5 -min & $\begin{array}{l}1.03- \\
13.59\end{array}$ & $2.55 \pm 0.73$ & Zhang et al. (2015) \\
\hline 5 high-elevation sites & Apr 2006 - May 2009 & 5 -min & & $1.65 \pm 0.32$ & Weiss-Penzias et al. (2015) \\
\hline $\begin{array}{l}\text { Mt. Front Lulin, Taiwan, } 23^{\circ} 30^{\prime} 36 " \mathrm{~N}, 120^{\circ} 55^{\prime} 12^{\prime \prime E} \\
(2862 \mathrm{~m})\end{array}$ & Mar, Jul, Sep, Dec 2008 & 5 -min & & $1.4-2.2$ & Weiss-Penzias et al. (2015) \\
\hline $\begin{array}{l}\text { Mt. Bachelor Observatory, Oregon, USA, } \\
43^{\circ} 58^{\prime} 48^{\prime \prime N}, 121^{\circ} 41^{\prime} 24^{\prime \prime}(2763 \mathrm{~m})\end{array}$ & $2006-2009$ & 5 -min & & $1.44-1.6$ & Weiss-Penzias et al. (2015) \\
\hline Reno, Nevada, USA, 39³4'12"N, 11947'W (1497m) & Jan 2005 - Aug 2007 & 5 -min & & $1.36-1.73$ & Weiss-Penzias et al. (2015) \\
\hline
\end{tabular}




\begin{tabular}{|l|l|l|l|l|}
\hline $\begin{array}{l}\text { Paradise Valley, Nevada, USA, } 41^{\circ} 30^{\prime} \mathrm{N}, 117^{\circ} 30^{\prime} \mathrm{W} \\
(1388 \mathrm{~m})\end{array}$ & Jun 2007 - Aug 2007 & 5 -min & Weiss-Penzias et al. (2015) & \\
\hline $\begin{array}{l}\text { Storm Peak Laboratory, Colorado, USA, } 40^{\circ} 27^{\prime} 36^{\prime \prime N}, \\
106^{\circ} 44^{\prime} 24^{\prime \prime} \mathrm{W}(3200 \mathrm{~m})\end{array}$ & May 2008 - Jun 2008 & 5 -min & & Weiss-Penzias et al. (2015) \\
\hline
\end{tabular}


Table S5: Continental boundary layer GOM $\left(\mathrm{pg} \mathrm{m}^{-3}\right)$ at remote, rural, urban, and high elevation sites. LOD indicates limit of detection.

\begin{tabular}{|c|c|c|c|c|c|}
\hline Location (elevation in m.a.s.l.) & Sampling period & $\begin{array}{l}\text { Time } \\
\text { resolution }\end{array}$ & Range & Mean $( \pm \sigma)$ & Reference \\
\hline \multicolumn{6}{|l|}{ Remote } \\
\hline $\begin{array}{l}\text { Experimental Lakes Area, Ontario, Canada, } \\
49^{\circ} 39^{\prime} 50^{\prime \prime} \mathrm{N}, 93^{\circ} 43^{\prime} 16^{\prime \prime} \mathrm{W}(369 \mathrm{~m})\end{array}$ & May 2005 - Dec 2006 & 3-h & \begin{tabular}{|l|}
$<$ LOD- \\
24.89 \\
\end{tabular} & $0.99 \pm 1.9$ & Cheng et al. (2012) \\
\hline Salmon Falls Creek Reservoir, Idaho, USA (1510m) & $\begin{array}{l}2 \text { weeks per season during summer } \\
2005 \text {-fall 2006; all of summer } \\
2006\end{array}$ & 1-h & & $3.2-8.1$ & Abbott et al. (2008) \\
\hline $\begin{array}{l}\text { Devil's Lake State Park, Wisconsin, USA, } 43^{\circ} 25^{\prime} 48^{\prime \prime N} \text {, } \\
89^{\circ} 40^{\prime} 48^{\prime \prime} \mathrm{W}(389 \mathrm{~m})\end{array}$ & Apr 2003 - Apr 2004 & $1-\mathrm{h}$ & $\begin{array}{l}<\text { LOD- } \\
151\end{array}$ & $3.8 \pm 8.9$ & Manolopoulos et al. (2007) \\
\hline $\begin{array}{l}\text { Devil's Lake State Park, Wisconsin, USA, } 43^{\circ} 26^{\prime} 5^{\prime \prime} \mathrm{N}, \\
89^{\circ} 40^{\prime} 48^{\prime \prime} \mathrm{W}\end{array}$ & Apr 2003 - Mar 2004 & 1-h & & $5.3 \pm 0.2$ & Rutter et al. (2008) \\
\hline Contiguous U.S. & Jan 2009 - Dec 2011 & 2-h & $0-223.5$ & $2.55 \pm 5.60$ & Gay et al. (2013) \\
\hline \multicolumn{6}{|l|}{ Rural } \\
\hline $\begin{array}{l}\text { Multiple sites in Canada, } 43^{\circ} 32^{\prime}-82^{\circ} 30^{\prime} \mathrm{N}, 114^{\circ} 12^{\prime} \text { - } \\
62^{\circ} 19^{\prime} \mathrm{W}\end{array}$ & $2002-2011$ & $1-3 \mathrm{~h}$ & & $0.5-100.9$ & Cole et al. (2014) \\
\hline $\begin{array}{l}\text { Huntington Forest, New York, USA, } 43^{\circ} 58^{\prime} 12^{\prime \prime} \mathrm{N}, \\
74^{\circ} 13^{\prime} 12^{\prime \prime} \mathrm{W}(500 \mathrm{~m})\end{array}$ & Jun 2006 - May 2007 & $1-\mathrm{h}$ & $\begin{array}{l}\text { <LOD- } \\
45.4\end{array}$ & $1.8 \pm 2.2$ & Choi et al. (2008) \\
\hline $\begin{array}{l}\text { Huntington Forest, New York, USA, } 43^{\circ} 58^{\prime} 12^{\prime \prime} \text {, } \\
74^{\circ} 13^{\prime} 12^{\prime \prime} \mathrm{W}(510 \mathrm{~m})\end{array}$ & Dec 2007 - Nov 2009 & 2-h & $\begin{array}{l}<\text { LOD- } \\
63.9\end{array}$ & $1.3 \pm 3.3$ & Choi et al. (2013) \\
\hline $\begin{array}{l}\text { Huntington Forest, New York, USA, } 43^{\circ} 58^{\prime} 12^{\prime \prime N} \text {, } \\
74^{\circ} 13^{\prime} 12^{\prime \prime} \mathrm{W}(500 \mathrm{~m})\end{array}$ & Jan 2009 - Dec 2010 & 2-h & $0-44$ & & Cheng et al. (2013) \\
\hline Maryhill, Ontario, Canada, $43^{\circ} 31^{\prime} \mathrm{N}, 80^{\circ} 25^{\prime} \mathrm{W}$ & Oct 2004 - Nov 2004 & 2-h & & $2.3 \pm 3.0$ & Cobbett and Van Heyst (2007) \\
\hline Atlanta, Georgia, USA & $2005-2008$ & $1-\mathrm{h}$ & & 8.6 & Nair et al. (2012) \\
\hline Wisconsin, USA, $43^{\circ} 26^{\prime} 24^{\prime \prime N}, 89^{\circ} 40^{\prime} 48^{\prime \prime} \mathrm{W}(389 \mathrm{~m})$ & 2003-2009 & 1-h & $\begin{array}{l}<\text { LOD- } \\
151\end{array}$ & $2.8-11.2$ & Engle et al. (2010) \\
\hline North Dakota, USA, $48^{\circ} 38^{\prime} 60^{\prime \prime} \mathrm{N}, 102^{\circ} 24^{\prime} \mathrm{W}(692 \mathrm{~m})$ & 2003-2009 & 1-h & $\begin{array}{l}<\text { LOD- } \\
19.2\end{array}$ & $2.0 \pm 2.6$ & Engle et al. (2010) \\
\hline 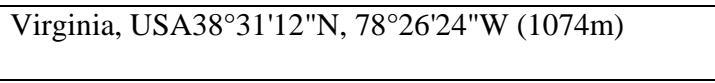 & $2003-2009$ & 1-h & \begin{tabular}{|l|}
$<$ LOD- \\
37.7 \\
\end{tabular} & $1.8 \pm 3.9$ & Engle et al. (2010) \\
\hline Dexter, Michigan, USA, $42^{\circ} 25^{\prime} 12^{\prime \prime} \mathrm{N}, 83^{\circ} 54^{\prime} \mathrm{W}$ & 2004 & 1-h & $1.0-121.7$ & $3.8 \pm 6.6$ & Liu et al. (2010) \\
\hline $\begin{array}{l}\text { San Francisco Bay Area, California, USA, } \\
37^{\circ} 11^{\prime} 24^{\prime \prime N}, 121^{\circ} 46^{\prime} 12^{\prime \prime W}(149 \mathrm{~m})\end{array}$ & $\begin{array}{l}9 \text { Sep } 2005-27 \text { Sep 2005, } 21 \text { Oct } \\
2008-30 \text { Oct } 2008\end{array}$ & 1-h & $0.2-154$ & $4.58-14.5$ & Rothenberg et al. (2010) \\
\hline Central Wisconsin, USA & Jun 2007 - Nov 2007 & 1-h & & $0.6-0.8$ & Kolker et al. (2010) \\
\hline Athens, Ohio, USA, $39^{\circ} 18^{\prime} \mathrm{N}, 82^{\circ} 7^{\prime} \mathrm{W}(330 \mathrm{~m})$ & Jul 2004 - Jul 2005 & 1-h & $0-461.59$ & $12.45 \pm 24.53$ & Yatavelli et al. (2006) \\
\hline Holland, Illinois, USA $42^{\circ} 46^{\prime} 4.8^{\prime \prime} \mathrm{N}, 86^{\circ} 8^{\prime} 56.4^{\prime \prime} \mathrm{W}$ & 1 Jul 2007 - 8 Nov 2007 & 1-h & $\leq 137$ & $8 \pm 15$ & Gratz et al. (2013) \\
\hline 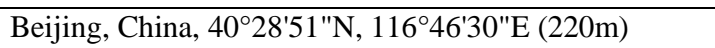 & Dec 2008 - Nov 2009 & 1-h & $0.12-$ & $10.1 \pm 18.8$ & Zhang et al. (2013) \\
\hline
\end{tabular}




\begin{tabular}{|c|c|c|c|c|c|}
\hline & & & 301.20 & & \\
\hline 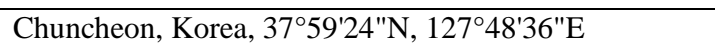 & $2006-2008$ & $24-h$ & $0.1-16.9$ & $2.7 \pm 2.7$ & Han et al. (2014) \\
\hline \multicolumn{6}{|l|}{ Urban } \\
\hline $\begin{array}{l}\text { Halifax, Nova Scotia, Canada, } 44^{\circ} 39^{\prime} 54.71^{\prime \prime N}, \\
63^{\circ} 34^{\prime} 9.69^{\prime \prime} \mathrm{W}(125 \mathrm{~m})\end{array}$ & Jan 2010 - Dec 2011 & $2-h$ & & $2.1 \pm 3.4$ & Cheng et al. (2014) \\
\hline Toronto, Ontario, Canada, $43^{\circ} 40^{\prime} \mathrm{N}, 7^{\circ} 24^{\prime} \mathrm{W}$ & Dec 2003 - Nov 2004 & 1-h & $\begin{array}{l}<\text { LOD- } \\
284\end{array}$ & $14.2 \pm 13.2$ & Song et al. (2009) \\
\hline Birmingham, Alabama, USA & $2005-2008$ & 1-h & & 78.2 & Nair et al. (2012) \\
\hline Reno, Nevada, USA, 39³4'12"N, 11947'W (1509m) & Nov 2004 - Nov 2007 & 2-h & $0-401$ & $26 \pm 35$ & Peterson et al. (2009) \\
\hline Illinois, USA, 38³6'36"N, 909'36"W (206m) & $2003-2009$ & 1-h & $\begin{array}{l}\text { <LOD- } \\
8160\end{array}$ & $11.6-63.3$ & Engle et al. (2010) \\
\hline Wisconsin, USA, $43^{\circ} 4^{\prime} 48^{\prime \prime} \mathrm{N}, 87^{\circ} 52^{\prime} 48^{\prime \prime} \mathrm{W}(184 \mathrm{~m})$ & 2003-2009 & 1-h & $\begin{array}{l}<\text { LOD- } \\
124\end{array}$ & $4.1-13.8$ & Engle et al. (2010) \\
\hline Detroit, Michigan, USA, $42^{\circ} 10^{\prime} 48^{\prime \prime} \mathrm{N}, 83^{\circ} 3^{\prime} 36^{\prime \prime} \mathrm{W}$ & 2004 & 1-h & $\begin{array}{l}1.0- \\
2472.9\end{array}$ & $15.5 \pm 54.9$ & Liu et al. (2010) \\
\hline $\begin{array}{l}\text { San Francisco Bay Area, California, USA, } 37^{\circ} 25^{\prime} 12^{\prime \prime N} \text {, } \\
122^{\circ} 2^{\prime} 24^{\prime \prime W}(11 \mathrm{~m})\end{array}$ & $\begin{array}{l}30 \text { Aug } 2009 \text { - } 9 \text { Sep } 2005,19 \text { Sep } \\
2008-30 \text { Sep } 2008\end{array}$ & 1-h & $0.2-19.5$ & $1.81-2.58$ & Rothenberg et al. (2010) \\
\hline $\begin{array}{l}\text { Rochester, New York, USA, } 43^{\circ} 8^{\prime} 46^{\prime \prime N}, 77^{\circ} 32^{\prime} 54^{\prime \prime} \mathrm{W} \\
(137 \mathrm{~m})\end{array}$ & $\begin{array}{l}\text { Dec } 2007-\text { Apr 2008; Dec } 2008- \\
\text { Apr } 2009\end{array}$ & $2-h$ & & 5.1 & Wang et al. (2013) \\
\hline $\begin{array}{l}\text { Rochester, New York, USA, } 43^{\circ} 8^{\prime} 46^{\prime \prime N}, 77^{\circ} 32^{\prime} 54^{\prime \prime W} \\
(137 \mathrm{~m})\end{array}$ & $\begin{array}{l}\text { Dec } 2007 \text { - Apr } 2008 \text { coal power } \\
\text { plant in operation }\end{array}$ & $2-\mathrm{h}$ & & 9.9 & Wang et al. (2013) \\
\hline $\begin{array}{l}\text { Rochester, New York, USA, } 43^{\circ} 8^{\prime} 46^{\prime \prime N}, 77^{\circ} 32^{\prime} 54^{\prime \prime} \mathrm{W} \\
(137 \mathrm{~m})\end{array}$ & $\begin{array}{l}\text { Dec } 2007 \text { - Apr } 2009 \text { coal power } \\
\text { plant shutdown }\end{array}$ & $2-\mathrm{h}$ & & 3.9 & Wang et al. (2013) \\
\hline 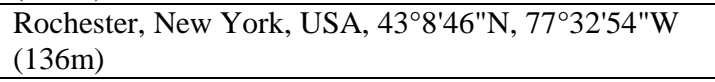 & Dec 2007 - Nov 2009 & $2-h$ & $\begin{array}{l}\text { LOD- } \\
177.6 \\
\end{array}$ & $5.6 \pm 10.3$ & Choi et al. (2013) \\
\hline 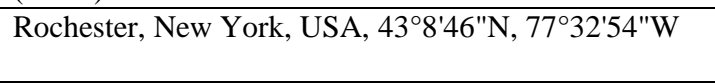 & Sep 2008 - Jun 2013 & $2-h$ & $\begin{array}{l}0.29- \\
23.25\end{array}$ & $8.24 \pm 12.94$ & Civerolo et al. (2014) \\
\hline New York City, USA, $40^{\circ} 52^{\prime} 05^{\prime \prime} \mathrm{N}, 73^{\circ} 52^{\prime} 42^{\prime \prime} \mathrm{W}$ & Sep 2008 - Jun 2013 & $2-h$ & $\begin{array}{l}0.78- \\
13.53\end{array}$ & $6.26 \pm 15.85$ & Civerolo et al. (2014) \\
\hline Milwaukee, Wisconsin, USA, $43^{\circ} 6^{\prime} 29^{\prime \prime N}, 87^{\circ} 53^{\prime} 2^{\prime \prime W}$ & Jun 2004 - May 2005 & 1-h & & $10.3 \pm 0.2$ & Rutter et al. (2008) \\
\hline $\begin{array}{l}\text { NV98, Nevada, USA, } 39^{\circ} 30^{\prime} 36^{\prime \prime} \mathrm{N}, 119^{\circ} 43^{\prime} 12^{\prime \prime} \mathrm{W} \\
(1340 \mathrm{~m})\end{array}$ & Jun 2007 - Aug 2007 & 2-h & $\begin{array}{l}<\text { LOD- } \\
157\end{array}$ & $46 \pm 32$ & Weiss-Penzias et al. (2009) \\
\hline DRI, Nevada, USA, 39³4'12"N,11947'W (1497m) & Jun 2007 - Aug 2007 & $2-\mathrm{h}$ & $5.7-401$ & $87 \pm 57$ & Weiss-Penzias et al. (2009) \\
\hline 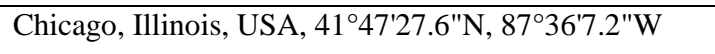 & 1 Jul 2007 - 8 Nov 2007 & 1-h & $\leq 2707$ & $17 \pm 87$ & Gratz et al. (2013) \\
\hline $\begin{array}{l}\text { Oxford, Mississippi, USA, } 34^{\circ} 21^{\prime} 50^{\prime \prime} \mathrm{N}, 89^{\circ} 32^{\prime} 60^{\prime \prime} \mathrm{W} \\
(150 \mathrm{~m})\end{array}$ & Jul 2011 - Jun 2012 & 1-h & $0-84$ & $3.87 \pm 6.17$ & Jiang et al. (2013) \\
\hline Contiguous U.S. & Jan 2009 - Dec 2011 & $2-h$ & $0-7694.8$ & $9.69 \pm 59.64$ & Gay et al. (2013) \\
\hline Mexico City, $19^{\circ} 29^{\prime} 21.4^{\prime \prime} \mathrm{N}, 9^{\circ} 8^{\prime} 51.8^{\prime \prime} \mathrm{W}$ & Mar 2006 & 1-h & & $62 \pm 64$ & Rutter et al. (2009) \\
\hline
\end{tabular}




\begin{tabular}{|c|c|c|c|c|c|}
\hline Göteborg, Sweden, $57^{\circ} 42^{\prime} \mathrm{N}, 11^{\circ} 54^{\prime} \mathrm{E}$ & Feb 2005 & 1-h & $\begin{array}{l}0.34- \\
32.84\end{array}$ & $2.53 \pm 4.09$ & Li et al. (2008) \\
\hline Seoul, Korea, $37^{\circ} 30^{\prime} 50.4^{\prime \prime N}, 127^{\circ} 0{ }^{\prime} 3.6^{\prime \prime} \mathrm{E}$ & $2006-2008$ & $24 \mathrm{~h}$ & $0.9-57.3$ & $11.3 \pm 9.5$ & Han et al. (2014) \\
\hline Fukuoka, Japan, $33^{\circ} 32^{\prime} 55^{\prime \prime N}, 130^{\circ} 21^{\prime} 52^{\prime \prime E}$ & Jun 2012 - May 2013 & $2-h$ & $\begin{array}{l}<\text { LOD- } \\
123\end{array}$ & $5.7 \pm 9.4$ & Marumoto et al. (2015) \\
\hline \multicolumn{6}{|l|}{ High elevation } \\
\hline $\begin{array}{l}\text { Pad Monadnock, New Hampshire, USA, 4251'36"N, } \\
71^{\circ} 52^{\prime} 48^{\prime \prime} \mathrm{W}(700 \mathrm{~m})\end{array}$ & 2007 & $2-\mathrm{h}$ & $0-33$ & $1.2 \pm 2.7$ & Sigler et al. (2009) \\
\hline $\begin{array}{l}\text { Pad Monadnock, New Hampshire, USA, } 42^{\circ} 51^{\prime} 36^{\prime N} \mathrm{~N}, \\
71^{\circ} 52^{\prime} 48^{\prime \prime} \mathrm{W}(700 \mathrm{~m})\end{array}$ & Jun 2005 - Oct 2008 & 90-min & $\begin{array}{l}<\text { LOD- } \\
5.1\end{array}$ & $0.18-1.4$ & Mao and Talbot (2012) \\
\hline $\begin{array}{l}\text { Look Rock, Tennessee, USA, } 35^{\circ} 36^{\prime} 36^{\prime \prime N}, 83^{\circ} 54^{\prime} 36^{\prime \prime W} \\
(813 \mathrm{~m})\end{array}$ & Spring-Summer 2004 & 1-h & & 5 & Valente et al. (2007) \\
\hline $\begin{array}{l}\text { Northern Nevada, USA, 4130' } 3.6^{\prime \prime} \mathrm{N}, 117^{\circ} 30^{\prime} 3.6^{\prime \prime} \mathrm{W} \\
\text { and } 41^{\circ} 33^{\prime} 3.6^{\prime \prime N}, 115^{\circ} 12^{\prime} 39.6^{\prime \prime} \mathrm{W}(1388-1806 \mathrm{~m})\end{array}$ & $\begin{array}{l}29 \text { Mar } 2005 \text { - } 4 \text { Apr 2005, } 26 \text { Sep } \\
2005 \text { - } 8 \text { Aug 2005, } 22 \text { Oct } 2005 \text { - } \\
28 \text { Oct 2005, 24 Jan } 2006-30 \\
\text { Jan2006 }\end{array}$ & 2-h & $\leq 150$ & $7.0-13.0$ & Lyman and Gustin (2008) \\
\hline NV02, Nevada, USA, $41^{\circ} 30^{\prime} \mathrm{N}, 117^{\circ} 30^{\prime} \mathrm{W}(1388 \mathrm{~m})$ & Jun 2007 - Aug 2007 & $2-\mathrm{h}$ & $\begin{array}{l}<\text { LOD- } \\
143\end{array}$ & $26 \pm 26$ & Weiss-Penzias et al. (2009) \\
\hline Storm Peak Laboratory, Colorado, USA (3220m) & Apr 2008 - Jul 2008 & 1-h & $0-137$ & $20 \pm 21$ & Faïn et al. (2009) \\
\hline $\begin{array}{l}\text { Mt. Bachelor Observatory, Oregon, USA, } 43^{\circ} 58^{\prime} 48^{\prime \prime N} \text {, } \\
121^{\circ} 41^{\prime} 24^{\prime \prime} \mathrm{W}(2700 \mathrm{~m})\end{array}$ & May 2005 - Aug 2005 & 3-h & $\begin{array}{l}<\text { LOD- } \\
141\end{array}$ & $39 \pm 75$ & Swartzendruber et al. (2006) \\
\hline $\begin{array}{l}\text { Contiguous U.S. high elevation sites and Mt. Lulin, } \\
\text { Taiwan }\end{array}$ & Jan 2009 - Dec 2011 & $2-\mathrm{h}$ & $0.2-555$ & $143.25 \pm 105.91$ & Gay et al. (2013) \\
\hline Waliguan, China, $36^{\circ} 17^{\prime} 12^{\prime \prime} \mathrm{N}, 100^{\circ} 53^{\prime} 52.7^{\prime \prime E},(3816 \mathrm{~m})$ & Sep 2007 - Sep 2008 & $3.5-7.5 \mathrm{~h}$ & & $7.4 \pm 4.8$ & Fu et al. (2012a) \\
\hline Mt. Changbai, China, $42^{\circ} 24^{\prime} \mathrm{N}, 128^{\circ} 28^{\prime} \mathrm{E}(738.1 \mathrm{~m})$ & Aug 2005 - Jul 2006 & 1-h & $0-880$ & $29-84$ & Wan et al. (2009b) \\
\hline Mt. Lulin, Taiwan, $23^{\circ} 30^{\prime} 36^{\prime \prime N}, 120^{\circ} 55^{\prime} 12^{\prime E}(2862 \mathrm{~m})$ & Apr 2006 - Sep 2007 & $1-2 \mathrm{~h}$ & $\leq 194.7$ & $12.1 \pm 20$ & Sheu et al. (2010) \\
\hline $\begin{array}{l}\text { Shangri-La, Tibetan Plateau, China, } 28^{\circ} 1^{\prime} \mathrm{N}, 9^{\circ} 44^{\prime} \mathrm{E} \\
(3580 \mathrm{~m})\end{array}$ & Nov 2009 - Nov 2010 & 1-h & $\begin{array}{l}0.71- \\
39.85 \\
\end{array}$ & $8.22 \pm 7.90$ & Zhang et al. (2015) \\
\hline 5 high-elevation sites & Apr 2006 - May 2009 & 2-h & & $\begin{array}{l}30 \pm 24 \\
(\mathrm{GOM}+\mathrm{PBM})\end{array}$ & Weiss-Penzias et al. (2015) \\
\hline $\begin{array}{l}\text { Mt. Front Lulin, Taiwan, } 23^{\circ} 30^{\prime} 36 " \mathrm{~N}, 120^{\circ} 55^{\prime} 12^{\prime \prime E} \\
(2862 \mathrm{~m})\end{array}$ & Mar, Jul, Sep, Dec 2008 & $2-\mathrm{h}$ & & $\begin{array}{l}11-27 \\
(\mathrm{GOM}+\mathrm{PBM})\end{array}$ & Weiss-Penzias et al. (2015) \\
\hline $\begin{array}{l}\text { Mt. Bachelor Observatory, Oregon, USA, } \\
43^{\circ} 58^{\prime} 48^{\prime \prime N}, 121^{\circ} 41^{\prime} 24^{\prime \prime}(2763 \mathrm{~m})\end{array}$ & $2006-2009$ & $2-\mathrm{h}$ & & $\begin{array}{l}32-44 \\
(\mathrm{GOM}+\mathrm{PBM})\end{array}$ & Weiss-Penzias et al. (2015) \\
\hline Reno, Nevada, USA, 39³4'12"N, 11947'W (1497m) & Jan 2005 - Aug 2007 & $2-\mathrm{h}$ & & $\begin{array}{l}14-68 \\
(\mathrm{GOM}+\mathrm{PBM})\end{array}$ & Weiss-Penzias et al. (2015) \\
\hline $\begin{array}{l}\text { Paradise Valley, Nevada, USA } 41^{\circ} 30^{\prime} \mathrm{N}, 117^{\circ} 30^{\prime} \mathrm{W} \\
(1388 \mathrm{~m})\end{array}$ & Jun 2007 - Aug 2007 & 2-h & & $\begin{array}{l}32 \pm 16 \\
(\mathrm{GOM}+\mathrm{PBM})\end{array}$ & Weiss-Penzias et al. (2015) \\
\hline $\begin{array}{l}\text { Storm Peak Laboratory, Colorado, USA, } 40^{\circ} 27^{\prime} 36^{\prime \prime} \mathrm{N}, \\
106^{\circ} 44^{\prime} 24^{\prime \prime} \mathrm{W}(3200 \mathrm{~m})\end{array}$ & May 2008 - Jun 2008 & 2-h & & $\begin{array}{l}27-34 \\
(\mathrm{GOM}+\mathrm{PBM})\end{array}$ & Weiss-Penzias et al. (2015) \\
\hline
\end{tabular}


Table S6: Continental boundary layer PBM $\left(\mathrm{pg} \mathrm{m}^{-3}\right)$ at remote, rural, urban, and high elevation sites. LOD indicates limit of detection; TPM indicates total particulate mercury; FPBM indicates fine particulate mercury; CPBM indicates coarse particulate mercury.

\begin{tabular}{|c|c|c|c|c|c|}
\hline Location (elevation in m.a.s.l.) & Sampling period & $\begin{array}{l}\text { Time } \\
\text { resolution }\end{array}$ & Range & Mean $( \pm \sigma)$ & Reference \\
\hline \multicolumn{6}{|l|}{ Remote } \\
\hline $\begin{array}{l}\text { Experimental Lakes Area, Ontario, Canada, } \\
49^{\circ} 39^{\prime} 50^{\prime \prime} \mathrm{N}, 93^{\circ} 43^{\prime} 16^{\prime \prime} \mathrm{W}(369 \mathrm{~m})\end{array}$ & May 2005 - Dec 2006 & 3-h & $\begin{array}{l}\text { <LOD- } \\
42.33\end{array}$ & $4.4 \pm 3.7$ & Cheng et al. (2012) \\
\hline $\begin{array}{l}\text { Devil's Lake State Park, Wisconsin, USA, } 43^{\circ} 25^{\prime} 48^{\prime \prime N} \text {, } \\
89^{\circ} 40^{\prime} 48^{\prime \prime} \mathrm{W}(389 \mathrm{~m})\end{array}$ & Apr 2003 - Apr 2004 & 1-h & $\begin{array}{l}<\text { LOD- } \\
205\end{array}$ & $8.6 \pm 8.3$ & Manolopoulos et al. (2007) \\
\hline $\begin{array}{l}\text { Devil's Lake State Park, Wisconsin, USA, } 43^{\circ} 26^{\prime} 5^{\prime \prime N} \text {, } \\
89^{\circ} 40^{\prime} 48^{\prime \prime} \mathrm{W}\end{array}$ & Apr 2003 - Mar 2004 & $1-\mathrm{h}$ & & $9.1 \pm 0.1$ & Rutter et al. (2008) \\
\hline Contiguous U.S. & Jan 2009 - Dec 2011 & $2-\mathrm{h}$ & $0-403.6$ & $5.12 \pm 7.43$ & Gay et al. (2013) \\
\hline \multicolumn{6}{|l|}{ Rural } \\
\hline $\begin{array}{l}\text { Multiple sites in Canada, } 43^{\circ} 32^{\prime}-82^{\circ} 30^{\prime} \mathrm{N}, 114^{\circ} 12^{\prime}- \\
62^{\circ} 19^{\prime} \mathrm{W}\end{array}$ & $2002-2011$ & $1-3 \mathrm{~h}$ & & $2.3-168.5$ & Cole et al. (2014) \\
\hline Maryhill, Ontario, Canada $43^{\circ} 31^{\prime} \mathrm{N}, 80^{\circ} 25^{\prime} \mathrm{W}$ & Oct 2004 - Nov 2004 & $2-\mathrm{h}$ & & $3.0 \pm 6.2$ & Cobbett and Van Heyst (2007) \\
\hline Atlanta, Georgia, USA & $2005-2008$ & 1-h & & 4.4 & Nair et al. (2012) \\
\hline Wisconsin, USA, $43^{\circ} 26^{\prime} 24^{\prime \prime} \mathrm{N}, 89^{\circ} 40^{\prime} 48^{\prime \prime} \mathrm{W}(389 \mathrm{~m})$ & 2003-2009 & $1-\mathrm{h}$ & $\begin{array}{l}<\text { LOD- } \\
205\end{array}$ & $6.4-12.2$ & Engle et al. (2010) \\
\hline North Dakota, USA, $48^{\circ} 38^{\prime} 60 " \mathrm{~N}, 102^{\circ} 24^{\prime} \mathrm{W}(692 \mathrm{~m})$ & 2003-2009 & $1-\mathrm{h}$ & $\begin{array}{l}\text { LOD- } \\
11.9\end{array}$ & $2.2 \pm 2.4$ & Engle et al. (2010) \\
\hline Virginia, USA38³1'12"N, 78²6'24"W (1074m) & $2003-2009$ & 1-h & $\begin{array}{l}\text { <LOD- } \\
19.4\end{array}$ & $4.6 \pm 3.9$ & Engle et al. (2010) \\
\hline Dexter, Michigan, USA, $42^{\circ} 25^{\prime} 12^{\prime \prime N}, 83^{\circ} 54^{\prime} \mathrm{W}$ & 2004 & 1-h & $1.0-90.6$ & $6.1 \pm 5.5$ & Liu et al. (2010) \\
\hline $\begin{array}{l}\text { Huntington Forest, New York, USA, } 43^{\circ} 58^{\prime} 12^{\prime \prime} \text {, } \\
74^{\circ} 13^{\prime} 12^{\prime \prime} \mathrm{W}(500 \mathrm{~m})\end{array}$ & Jun 2006 - May 2007 & 1-h & $\begin{array}{l}<\text { LOD- } \\
54.0\end{array}$ & $3.2 \pm 3.7$ & Choi et al. (2008) \\
\hline $\begin{array}{l}\text { Huntington Forest, New York, USA, } 43^{\circ} 58^{\prime} 12^{\prime \prime} \text {, } \\
74^{\circ} 13^{\prime} 12^{\prime \prime} \mathrm{W}(510 \mathrm{~m})\end{array}$ & Dec 2007 - Nov 2009 & $2-h$ & $\begin{array}{l}\text { <LOD- } \\
97.6\end{array}$ & $4.1 \pm 7.8$ & Choi et al. (2013) \\
\hline $\begin{array}{l}\text { Huntington Forest, New York, USA, } 43^{\circ} 58^{\prime} 12^{\prime \prime N} \text {, } \\
74^{\circ} 13^{\prime} 12^{\prime \prime} \mathrm{W}(500 \mathrm{~m})\end{array}$ & Jan 2009 - Dec 2010 & $2-h$ & $0-39$ & & Cheng et al. (2013) \\
\hline $\begin{array}{l}\text { San Francisco Bay Area, California, USA, } \\
37^{\circ} 11^{\prime} 24^{\prime \prime N}, 121^{\circ} 46^{\prime} 12^{\prime \prime} \mathrm{W}(149 \mathrm{~m})\end{array}$ & $\begin{array}{l}\text { 9 Sep 2005-27 Sep 2005, } 21 \text { Oct } \\
\text { 2008-30 Oct } 2008\end{array}$ & 1-h & $0.2-37.5$ & $3.68-7.99$ & Rothenberg et al. (2010) \\
\hline Central Wisconsin, USA & Jun 2007 - Nov 2007 & $1-\mathrm{h}$ & & $2.6-5.0$ & Kolker et al. (2010) \\
\hline Athens, Ohio, USA, $39^{\circ} 18^{\prime} \mathrm{N}, 82^{\circ} 7^{\prime} \mathrm{W}(330 \mathrm{~m})$ & Jul 2004 - Jul 2005 & 1-h & $0-76.82$ & $5.29 \pm 6.04$ & Yatavelli et al. (2006) \\
\hline Holland, Illinois, USA $42^{\circ} 46^{\prime} 4.8^{\prime \prime} \mathrm{N}, 86^{\circ} 8^{\prime} 56.4^{\prime \prime} \mathrm{W}$ & 1 Jul 2007 - 8 Nov 2007 & 1-h & $\leq 31$ & $6 \pm 4$ & Gratz et al. (2013) \\
\hline Lichwin, Poland, $49^{\circ} 52.957^{\prime} \mathrm{N}, 20^{\circ} 57.024^{\prime} \mathrm{E}$ & 19 Aug 2003 - 29 Aug 2003 & $23-\mathrm{h}$ & $\begin{array}{l}0.02-0.2 \\
\mathrm{ng} \mathrm{m}^{-3} \\
\text { TPM }\end{array}$ & $\begin{array}{l}0.11 \pm 0.05 \mathrm{ng} \\
\mathrm{m}^{-3} \mathrm{TPM}\end{array}$ & Zielonka et al. (2005) \\
\hline
\end{tabular}




\begin{tabular}{|c|c|c|c|c|c|}
\hline Lichwin, Poland, $49^{\circ} 52.957^{\prime} \mathrm{N}, 20^{\circ} 57.024^{\prime} \mathrm{E}$ & 6 Jan 2004 - 3 Feb 2004 & $23-\mathrm{h}$ & & $1.05 \pm 0.18$ & Zielonka et al. (2005) \\
\hline Jeziory, Poland & Apr 2013 - Oct 2014 & $24-72 \mathrm{~h}$ & $\begin{array}{l}\text { FPBM: } \\
\text { <LOD- } \\
16.1, \\
\text { CPBM: } \\
\text { <LOD- } \\
142.5\end{array}$ & $\begin{array}{l}\text { FPBM: } \\
2.4 \pm 2.8 \text {, } \\
\text { CPBM: } \\
20.8 \pm 21.6\end{array}$ & Siudek et al. (2016) \\
\hline 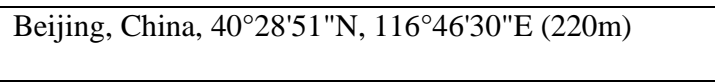 & Dec 2008 - Nov 2009 & 1-h & $\begin{array}{l}0.49- \\
1090.24\end{array}$ & $98.2 \pm 112.7$ & Zhang et al. (2013) \\
\hline Chuncheon, Korea, 3759'24"N, 12748'36"E & $\begin{array}{l}\text { 2006-2008; Fall 2009, winter } \\
\text { 2009, summer } 2010 \text { for TPM }\end{array}$ & $24-48 \mathrm{~h}$ & $0.1-30$ & $\begin{array}{l}3.7 \pm 5.7 \\
\text { TPM: } 0.9- \\
8.5\end{array}$ & Kim et al. (2012); Han et al. (2014) \\
\hline \multicolumn{6}{|l|}{ Urban } \\
\hline $\begin{array}{l}\text { Halifax, Nova Scotia, Canada, } 44^{\circ} 39^{\prime} 54.71^{\prime \prime N} \text {, } \\
63^{\circ} 34^{\prime} 9.69^{\prime \prime} \mathrm{W}(125 \mathrm{~m})\end{array}$ & Jan 2010 - Dec 2011 & $2-h$ & & $2.3 \pm 3.1$ & Cheng et al. (2014) \\
\hline Toronto, Ontario, Canada, $43^{\circ} 40^{\prime} \mathrm{N}, 7^{\circ} 24^{\prime} \mathrm{W}$ & Dec 2003 - Nov 2004 & 1-h & $\begin{array}{l}<\text { LOD- } \\
252\end{array}$ & $21.5 \pm 16.4$ & Song et al. (2009) \\
\hline Birmingham, Alabama, USA & $2005-2008$ & 1-h & & 39.5 & Nair et al. (2012) \\
\hline Reno, Nevada, USA, $39^{\circ} 34^{\prime} 12^{\prime \prime N}, 119^{\circ} 47^{\prime} \mathrm{W}$ (1509m) & Nov 2004 - Nov 2007 & $2-\mathrm{h}$ & $0-180$ & $9 \pm 10$ & Peterson et al. (2009) \\
\hline Illinois, USA, 38³6'36"N, 909'36"W (206m) & 2003-2009 & 1-h & $\begin{array}{l}\text { LOD- } \\
11600\end{array}$ & $12.9-37.9$ & Engle et al. (2010) \\
\hline Wisconsin, USA, $43^{\circ} 4^{\prime} 48^{\prime \prime} \mathrm{N}, 87^{\circ} 52^{\prime} 48^{\prime \prime W}(184 \mathrm{~m})$ & 2003-2009 & 1-h & $\begin{array}{l}<\text { LOD- } \\
507\end{array}$ & $10.2-15.7$ & Engle et al. (2010) \\
\hline Detroit, Michigan, USA, $42^{\circ} 10^{\prime} 48^{\prime \prime} \mathrm{N}, 83^{\circ} 3^{\prime} 36^{\prime \prime} \mathrm{W}$ & 2004 & 1-h & $1.0-1345.2$ & $18.1 \pm 61$ & Liu et al. (2010) \\
\hline $\begin{array}{l}\text { San Francisco Bay Area, California, USA, } 37^{\circ} 25^{\prime} 12^{\prime \prime N} \text {, } \\
122^{\circ} 2^{\prime} 24^{\prime \prime} \mathrm{W}(11 \mathrm{~m})\end{array}$ & $\begin{array}{l}30 \text { Aug } 2009 \text { - } 9 \text { Sep 2005, } 19 \text { Sep } \\
2008 \text { - } 30 \text { Sep } 2008\end{array}$ & 1-h & $0.2-29.4$ & $3.06-3.17$ & Rothenberg et al. (2010) \\
\hline $\begin{array}{l}\text { Rochester, New York, USA, } 43^{\circ} 8^{\prime} 46^{\prime \prime N}, 7^{\circ} 32^{\prime} 54^{\prime \prime} \mathrm{W} \\
(137 \mathrm{~m})\end{array}$ & $\begin{array}{l}\text { Dec } 2007 \text { - Apr 2008; Dec } 2008- \\
\text { Apr } 2009\end{array}$ & $2-h$ & & 8.9 & Wang et al. (2013) \\
\hline 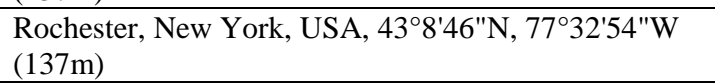 & $\begin{array}{l}\text { Dec } 2007 \text { - Apr } 2008 \text { coal power } \\
\text { plant in operation }\end{array}$ & $2-h$ & & 21.8 & Wang et al. (2013) \\
\hline 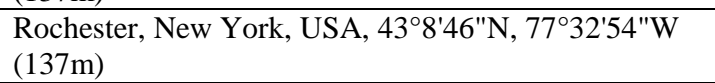 & $\begin{array}{l}\text { Dec } 2007 \text { - Apr } 2009 \text { coal power } \\
\text { plant shutdown }\end{array}$ & $2-\mathrm{h}$ & & 7.3 & Wang et al. (2013) \\
\hline 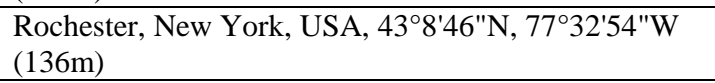 & Dec 2007 - Nov 2009 & $2-h$ & $\begin{array}{l}<\text { LOD- } \\
271.2\end{array}$ & $8.7 \pm 12.8$ & Choi et al. (2013) \\
\hline 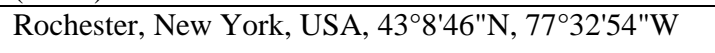 & Sep 2008 - Jun 2013 & $2-h$ & $2.36-27.32$ & $13.48 \pm 21.01$ & Civerolo et al. (2014) \\
\hline New York City, USA, $40^{\circ} 52^{\prime} 05^{\prime \prime} \mathrm{N}, 73^{\circ} 52^{\prime} 42^{\prime \prime} \mathrm{W}$ & Sep 2008 - Jun 2013 & $2-h$ & $1.42-15.82$ & $7.96 \pm 12.13$ & Civerolo et al. (2014) \\
\hline 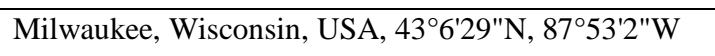 & Jun 2004 - May 2005 & 1-h & & $11.8 \pm 0.3$ & Rutter et al. (2008) \\
\hline $\begin{array}{l}\text { NV98, Nevada, USA, } 39^{\circ} 30^{\prime} 36^{\prime \prime N}, 119^{\circ} 43^{\prime} 12^{\prime \prime} \mathrm{W} \\
(1340 \mathrm{~m})\end{array}$ & Jun 2007 - Aug 2007 & $2-h$ & $<\mathrm{LOD}-55$ & $5 \pm 4$ & Weiss-Penzias et al. (2009) \\
\hline DRI, Nevada, USA, 39³4'12"N,11947'W (1497m) & Jun 2007 - Aug 2007 & 2-h & $<$ LOD- & $10 \pm 10$ & Weiss-Penzias et al. (2009) \\
\hline
\end{tabular}




\begin{tabular}{|c|c|c|c|c|c|}
\hline & & & 180 & & \\
\hline Chicago, Illinois, USA, $41^{\circ} 47^{\prime} 27.6^{\prime \prime} \mathrm{N}, 87^{\circ} 36^{\prime} 7.2^{\prime \prime} \mathrm{W}$ & 1 Jul 2007 - 8 Nov 2007 & 1-h & $\leq 401$ & $9 \pm 20$ & Gratz et al. (2013) \\
\hline $\begin{array}{l}\text { Oxford, Mississippi, USA, 34²1'50"N, 89³2'60"W } \\
(150 \mathrm{~m})\end{array}$ & Jul 2011 - Jun 2012 & 1-h & $0-33$ & $4.58 \pm 3.40$ & Jiang et al. (2013) \\
\hline Contiguous U.S. & Jan 2009 - Dec 2011 & $2-h$ & $0-3687.2$ & $8.46 \pm 29.05$ & Gay et al. (2013) \\
\hline Mexico City, $19^{\circ} 29^{\prime} 21.4^{\prime \prime} \mathrm{N}, 99^{\circ} 8^{\prime} 51.8^{\prime \prime} \mathrm{W}$ & Mar 2006 & 1-h & & $187 \pm 300$ & Rutter et al. (2009) \\
\hline São Paulo State, Brazil & $2002-2003$ & 24-h & & $\begin{array}{l}\text { TPM: } \\
400 \pm 300\end{array}$ & Fostier and Michelazzo, (2006) \\
\hline Göteborg, Sweden, $57^{\circ} 42^{\prime} \mathrm{N}, 11^{\circ} 54^{\prime} \mathrm{E}$ & Feb 2005 & 1-h & $3.89-20.26$ & $12.5 \pm 5.88$ & Li et al. (2008) \\
\hline Poznań, Poland, $52^{\circ} 42^{\prime} \mathrm{N}, 16^{\circ} 88^{\prime} \mathrm{E}$ & Apr 2013 - Oct 2014 & $24-72 \mathrm{~h}$ & $\begin{array}{l}\text { FPBM: } \\
\text { <LOD- } \\
77.1, \\
\text { CPBM: } \\
\text { <LOD- } \\
604.9\end{array}$ & $\begin{array}{l}\text { FPBM: } \\
7.3 \pm 9.1 \\
\text { CPBM: } \\
22.6 \pm 45.3\end{array}$ & Siudek et al. (2016) \\
\hline Shanghai, China & Jul 2004 - Apr 2006 & $24-h$ & $\begin{array}{l}0.07-1.45 \\
\text { ng m}^{-3} \\
\text { TPM }\end{array}$ & $\begin{array}{l}0.56 \pm 0.22 \mathrm{ng} \\
\mathrm{m}^{-3} \mathrm{TPM}\end{array}$ & Xiu et al. (2009) \\
\hline Shanghai, China & Jul 2004 - Apr 2006 & $24-h$ & $\begin{array}{l}0.2-0.47 \\
\mathrm{ng} \mathrm{m}^{-3} \\
\mathrm{TPM} \\
\end{array}$ & $\begin{array}{l}0.33 \pm 0.09 \mathrm{ng} \\
\mathrm{m}^{-3} \mathrm{TPM}\end{array}$ & Xiu et al. (2009) \\
\hline Beijing, China & Jan 2006 - Dec 2006 & $12-h$ & & $\begin{array}{l}263 \pm 246 \\
\text { (day) } \\
280 \pm 383 \\
\text { (night) } \\
\end{array}$ & Schleicher et al. (2015) \\
\hline Seoul, Korea, $37^{\circ} 30^{\prime} 50.4^{\prime \prime N}, 127^{\circ} 0^{\prime} 3.6^{\prime \prime E}$ & $\begin{array}{l}\text { 2006-2008; winter, summer } 2010 \\
\text { for TPM }\end{array}$ & $24-48 \mathrm{~h}$ & $2.1-64.3$ & $\begin{array}{l}13.4 \pm 12.0, \\
\text { TPM: } 1.1- \\
18.9\end{array}$ & Kim et al. (2012); Han et al. (2014) \\
\hline Fukuoka, Japan, $33^{\circ} 32^{\prime} 55^{\prime \prime N}, 130^{\circ} 21^{\prime} 52^{\prime \prime E}$ & Jun 2012 - May 2013 & $2-h$ & $\begin{array}{l}<\text { LOD- } \\
139\end{array}$ & $10 \pm 11$ & Marumoto et al. (2015) \\
\hline \multicolumn{6}{|l|}{ High elevation } \\
\hline $\begin{array}{l}\text { Look Rock, Tennessee, USA, 35³6'36"N, 8354'36"W } \\
(813 \mathrm{~m})\end{array}$ & Spring-Summer 2004 & 1-h & & 7 & Valente et al. (2007) \\
\hline $\begin{array}{l}\text { Northern Nevada, USA, 41 }{ }^{\circ} 30^{\prime} 3.6^{\prime \prime} \mathrm{N}, 117^{\circ} 30^{\prime} 3.6^{\prime \prime} \mathrm{W} \\
\text { and } 41^{\circ} 33^{\prime} 3.6^{\prime \prime} \mathrm{N}, 115^{\circ} 12^{\prime} 39.6^{\prime \prime} \mathrm{W}(1388-1806 \mathrm{~m})\end{array}$ & $\begin{array}{l}29 \text { Mar } 2005 \text { - } 4 \text { Apr 2005, } 26 \text { Sep } \\
2005-8 \text { Aug 2005, 22 Oct } 2005- \\
28 \text { Oct 2005, 24 Jan } 2006-30 \\
\text { Jan2006 }\end{array}$ & $2-h$ & $\leq 65$ & $9.0-13.0$ & Lyman and Gustin (2008) \\
\hline NV02, Nevada, USA, $41^{\circ} 30^{\prime} \mathrm{N}, 117^{\circ} 30^{\prime} \mathrm{W}(1388 \mathrm{~m})$ & Jun 2007 - Aug 2007 & $2-h$ & $\begin{array}{l}<\text { LOD- } \\
102\end{array}$ & $6 \pm 9$ & Weiss-Penzias et al. (2009) \\
\hline Storm Peak Laboratory, Colorado, USA (3220m) & Apr 2008 - Jul 2008 & 1-h & $0-33$ & $9 \pm 6$ & Faïn et al. (2009) \\
\hline
\end{tabular}




\begin{tabular}{|c|c|c|c|c|c|}
\hline $\begin{array}{l}\text { Mt. Bachelor Observatory, Oregon, USA, } 43^{\circ} 58^{\prime} 48^{\prime \prime} \mathrm{N} \text {, } \\
121^{\circ} 41^{\prime} 24^{\prime \prime} \mathrm{W}(2700 \mathrm{~m})\end{array}$ & May 2005 - Aug 2005 & $3-\mathrm{h}$ & $<$ LOD-11 & $4.4 \pm 4.4$ & Swartzendruber et al. (2006) \\
\hline $\begin{array}{l}\text { Contiguous U.S. high elevation sites and Mt. Lulin, } \\
\text { Taiwan }\end{array}$ & Jan 2009 - Dec 2011 & $2-h$ & $0-555.6$ & $90.69 \pm 92.07$ & Gay et al. (2013) \\
\hline Waliguan, China, $36^{\circ} 17^{\prime} 12^{\prime \prime N}, 100^{\circ} 53^{\prime} 52.7^{\prime \prime E},(3816 \mathrm{~m})$ & Sep 2007 - Sep 2008 & $3.5-7.5 \mathrm{~h}$ & & $19.4 \pm 18.1$ & Fu et al. (2012a) \\
\hline Mt. Changbai, China, $42^{\circ} 24^{\prime} \mathrm{N}, 128^{\circ} 28^{\prime} \mathrm{E}(738.1 \mathrm{~m})$ & Aug 2005 - Jul 2006 & $1-\mathrm{h}$ & $0-1001$ & $15-167$ & Wan et al. (2009b) \\
\hline Mt. Lulin, Taiwan, $23^{\circ} 30^{\prime} 36^{\prime \prime N}, 120^{\circ} 55^{\prime} 12^{\prime \prime E ~(2862 m) ~}$ & Apr 2006 - Sep 2007 & $1-2 \mathrm{~h}$ & 38.4 & $2.3 \pm 3.9$ & Sheu et al. (2010) \\
\hline $\begin{array}{l}\text { Shangri-La, Tibetan Plateau, China, } 28^{\circ} 1^{\prime} \mathrm{N}, 99^{\circ} 44^{\prime} \mathrm{E} \\
(3580 \mathrm{~m})\end{array}$ & Nov 2009 - Nov 2010 & 1-h & $\begin{array}{l}3.02- \\
185.59\end{array}$ & $38.32 \pm 31.26$ & Zhang et al. (2015) \\
\hline
\end{tabular}


Table S7. Airborne measurements of TGM/GEM $\left(\mathrm{ng} \mathrm{m}^{-3}\right)$ and GOM/PBM $\left(\mathrm{pg} \mathrm{m}^{-3}\right)$, units stated otherwise.

\begin{tabular}{|c|c|c|c|c|c|c|}
\hline Region & Flight domain & Time Period & Altitude & Hg species & Range & Reference \\
\hline Atlantic & $\begin{array}{l}\text { Cologne, via Greenland, } \\
\text { Canada, San Francisco, to } \\
\text { Putna Arena, Chile } \\
\end{array}$ & 1977 & $8 \mathrm{~km}$ & TGM & $\begin{array}{l}\text { SH: } 1.08 \pm 0.36 \\
\text { NH: } 1.45 \pm 0.22 \\
\text { ITCZ: } 2.4-2.7 \\
\end{array}$ & Seiler et al. (1980) \\
\hline Central Europe & -- & $\begin{array}{l}\text { Jun \& Aug } 1977- \\
\text { 1979; Feb } 1980\end{array}$ & $6-8 \mathrm{~km}$ & TGM & $2.24 \pm 0.51$ & Slemr et al. (1985) \\
\hline Germany & $400 \mathrm{~km}$ btw Munich and Halle & 13 Jun 1996 & $\begin{array}{l}0.9 \mathrm{~km} \\
2.5 \mathrm{~km}\end{array}$ & TGM & $\begin{array}{l}1.774 \pm 0.101 \\
1.635 \pm 0.094 \\
\end{array}$ & $\begin{array}{l}\text { Ebinghaus \& } \\
\text { Slemr }(2000) \\
\end{array}$ \\
\hline Barrow, AK & Northeast of Point Barrow & Mar-Apr 2000 & $\begin{array}{l}100 \mathrm{~m} \\
1000 \mathrm{~m}\end{array}$ & GOM & $\begin{array}{l}20 \\
2\end{array}$ & $\begin{array}{l}\text { Lindberg et al. } \\
(2002)\end{array}$ \\
\hline Canada & $\begin{array}{l}\text { Southeastern } \\
\text { Southern \& central ON } \\
\text { Arctic }\end{array}$ & $\begin{array}{l}\text { Summer 1995, } 97 \\
\text { Winter 1997-98 } \\
\text { Spring } 1998 \\
\end{array}$ & $0.1-7 \mathrm{~km}$ & GEM & $\begin{array}{l}1.5 \\
1.7 \\
1.7(>1 \mathrm{~km}) ; 1.2(<1 \mathrm{~km}) \\
\end{array}$ & Banic et al. (2003) \\
\hline East Asia & $24^{\circ} \mathrm{N}-44^{\circ} \mathrm{N}, 124^{\circ} \mathrm{E}-146^{\circ} \mathrm{E}$ & April 2001 & $\mathrm{SL}-7 \mathrm{~km}$ & $\begin{array}{l}\text { TGM } \\
\text { PBM } \\
\end{array}$ & $\begin{array}{l}\text { Mean: } 1.68(2.5-2 \mathrm{~km})- \\
2.13(0-0.5 \mathrm{~km}) \\
14-917\end{array}$ & $\begin{array}{l}\text { Friedli et al. } \\
(2004)\end{array}$ \\
\hline South America & $\begin{array}{l}\text { Btw São Paulo and Santiago } \\
\text { de Chile, } 25^{\circ} \mathrm{S}-50^{\circ} \mathrm{N} \\
\text { Btw Frankfurt and São Paulo }\end{array}$ & $\begin{array}{l}31 \text { Aug, } 5 \text { Oct } \\
2005 \\
4 \text { Oct } 2005\end{array}$ & $\mathrm{SL}-11 \mathrm{~km}$ & GEM & $\begin{array}{l}\text { NH: } 1.70-2.21 \\
\text { SH: } 1.35-\sim 1.7\end{array}$ & $\begin{array}{l}\text { Ebinghaus et al. } \\
\text { (2007) }\end{array}$ \\
\hline $\begin{array}{l}\text { North Pacific } \\
\text { Coastal CA\&WA }\end{array}$ & $35^{\circ} \mathrm{N}-55^{\circ} \mathrm{N}$ & Spring 2002 & $0-8 \mathrm{~km}$ & GEM & Median: $1.2-0.5$ & $\begin{array}{l}\text { Radke et al. } \\
(2007)\end{array}$ \\
\hline Pacific Northwest & $46^{\circ} \mathrm{N}-49^{\circ} \mathrm{N}, 125^{\circ} \mathrm{W}-121^{\circ} \mathrm{W}$ & $\begin{array}{l}12 \text { Apr - 15 May } \\
2006\end{array}$ & Surface $-6 \mathrm{~km}$ & GEM & $1.43 \pm 0.166 ; 0.994-2.02$ & $\begin{array}{l}\text { Swatzendruber et } \\
\text { al. (2008) }\end{array}$ \\
\hline West Pacific & $\begin{array}{l}\text { Honolulu, HW; Anchorage, } \\
\text { AL; Mexico City }\end{array}$ & $\begin{array}{l}\text { 4 Mar - 12 May } \\
2006\end{array}$ & Surface $-12 \mathrm{~km}$ & GEM & $0-\sim 250 \mathrm{ppqv}$ & $\begin{array}{l}\text { Talbot et al. } \\
(2007,2008)\end{array}$ \\
\hline $\begin{array}{l}\text { North America \& } \\
\text { Europe }\end{array}$ & $40^{\circ} \mathrm{N}-44^{\circ} \mathrm{N}, 75^{\circ} \mathrm{W}-100^{\circ} \mathrm{W}$ & 5 Nov 2011 & $\sim 6 \mathrm{~km}$ & $\begin{array}{l}\text { GEM } \\
\text { GOM }\end{array}$ & $\begin{array}{l}>0.3-\sim 1.3 \\
0-600\end{array}$ & $\begin{array}{l}\text { Lyman \& Jaffe } \\
(2012)\end{array}$ \\
\hline $\begin{array}{l}\text { Tullahoma, } \\
\text { Tennessee, USA }\end{array}$ & -- & $\begin{array}{l}\text { Aug } 2012-\text { Jun } \\
2013\end{array}$ & $0-6 \mathrm{~km}$ & $\begin{array}{l}\text { GEM } \\
\text { GOM } \\
\text { PBM }\end{array}$ & $\begin{array}{l}1.38 \pm 0.17 ; 0.75-2.05 \\
34.8 \pm 28.9 ; 0-20 \text { (win); } \\
<125 \text { (sum) } \\
29.6 \pm 29.5 ; 4.4-194.9\end{array}$ & $\begin{array}{l}\text { Brooks et al. } \\
(2014)\end{array}$ \\
\hline Southeastern US & $\begin{array}{l}\text { Flights over } 32^{\circ} \mathrm{N}- \\
36^{\circ} \mathrm{N}, 122^{\circ} \mathrm{W}-77^{\circ} \mathrm{W}\end{array}$ & $\begin{array}{l}1 \text { Jun - 15 Jul } \\
2013\end{array}$ & -- & $\begin{array}{l}\mathrm{THg} \\
\text { GOM }\end{array}$ & $\begin{array}{l}1.49 \pm 0.16 \\
212 \pm 112 ; \text { up to } 300-680\end{array}$ & $\begin{array}{l}\text { Gratz et al. (2015) } \\
\text { Shah et al. (2016) }\end{array}$ \\
\hline
\end{tabular}




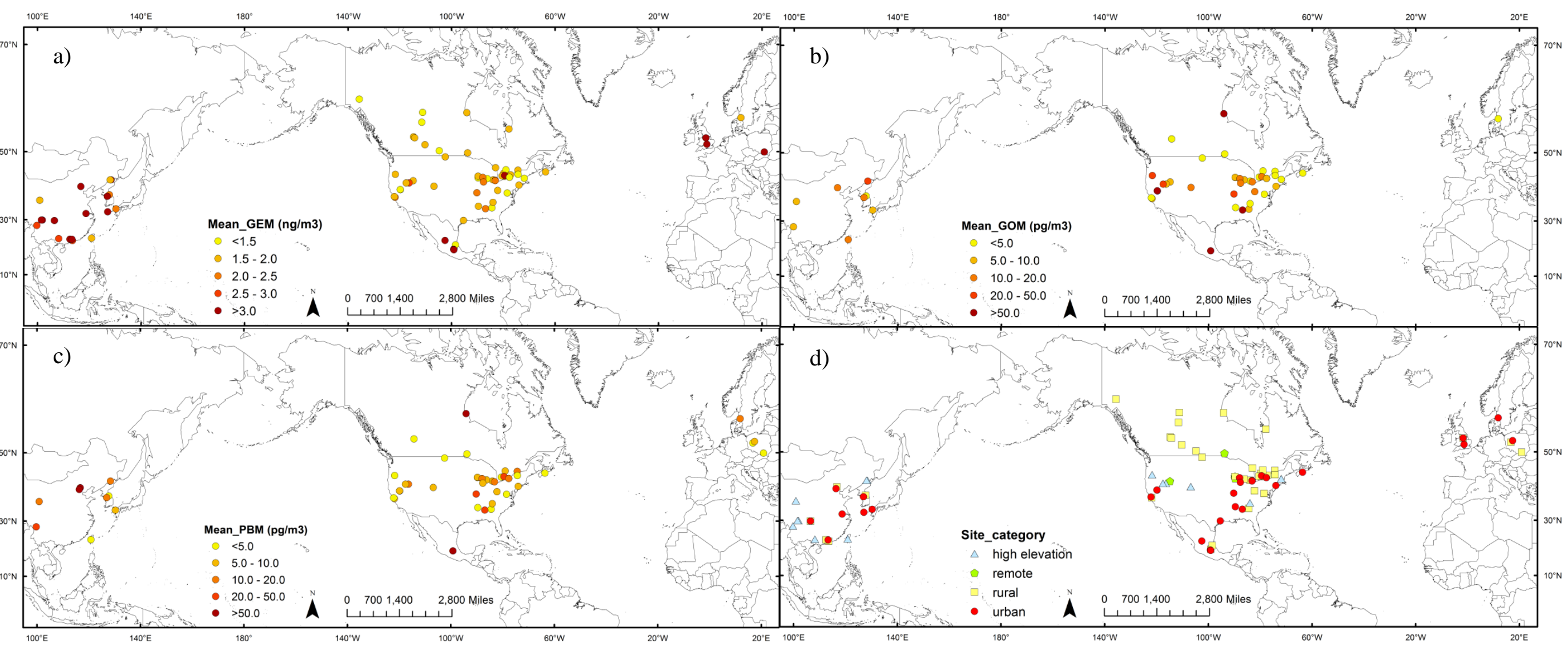

Figure S1. Maps of mean TGM/GEM (a), GOM (b) and PBM (c) concentrations at the continental sites (d) as listed in Tables S4S6. 


\section{References cited in SI not in the main text:}

Chen, L., Zhang, Y., Jacob, D. J., Soerensen, A. L., Fisher, J. A., Horowitz, H. M., Corbitt, E. S., and Wang, X.:, A decline in Arctic Ocean mercury suggested by differences in decadal trends of atmospheric mercury between the Arctic and northern midlatitudes, Geophys. Res. Lett., 42, 6076-6083, doi:10.1002/2015GL064051, 2015.

Cheng, I., Zhang, L., Blanchard, P., Dalziel, J., Tordon, R., Huang, J., and Holsen, T. M.: Comparisons of mercury sources and atmospheric mercury processes between a coastal and inland site, J. Geophys. Res.-Atmos., 118, 2434-2443, 2013.

Cheng, I., Zhang, L., Mao, H., Blanchard, P., Tordon, R., and Dalziel, J.: Seasonal and diurnal patterns of speciated atmospheric mercury at a coastal-rural and a coastal-urban site, Atmos. Environ., 82, 193-205, 2014.

Gay, D. A., Schmeltz, D., Prestbo, E., Olson, M., Sharac, T., and Tordon, R.: The Atmospheric Mercury Network: measurement and initial examination of an ongoing atmospheric mercury record across North America, Atmos. Chem. Phys., 13, 11339-11349, doi:10.5194/acp-13-11339-2013, 2013.

Kolker, A., Olson, M. L., Krabbenhoft, D. P., Tate, M. T., and Engle, M. A.: Patterns of mercury dispersion from local and regional emission sources, rural Central Wisconsin, USA, Atmos. Chem. Phys., 10, 4467-4476, doi:10.5194/acp-10-4467-2010, 2010.

Rutter, A. P., Snyder, D. C., Stone, E. A., Schauer, J. J., Gonzalez-Abraham, R., Molina, L. T., Márquez, C., Cárdenas, B., and de Foy, B.: In situ measurements of speciated atmospheric mercury and the identification of source regions in the Mexico City Metropolitan Area, Atmos. Chem. Phys., 9, 207-220,doi:10.5194/acp-9-207-2009, 2009.

Siudek, P., Frankowski, M., and Siepak, J.: Atmospheric particulate 50 mercury at the urban and forest sites in central Poland, Environ. Sci. Pollut. Res., 23, 2341-2352, 2016.

Valente, R. J., Shea, C., Humes, K. L., and Tanner, R. L.: Atmospheric mercury in the Great Smoky Mountains compared to regional and global levels, Atmos. Environ., 41, 1861-1873, 2007.

Yang, Y., Chen, H., and Wang, D.: Spatial and temporal distribution of gaseous elemental mercury in Chongqing, China, Environ. Monit. Assess., 156, 479-489, 2009. 